\title{
Response of Oceanic Hydrate-Bearing Sediments to Thermal Stresses
}

\author{
G.J. Moridis, SPE, and M. B. Kowalsky, Lawrence Berkeley National Laboratory
}

\begin{abstract}
In this study we evaluate the response of oceanic subsurface systems to thermal stresses caused by the flow of warm fluids through non-insulated well systems crossing hydrate-bearing sediments. Heat transport from warm fluids, originating from deeper reservoirs under production, into the geologic media can cause dissociation of the gas hydrates. The objective of this study is to determine whether gas evolution from hydrate dissociation can lead to excessive pressure build-up and possibly to fracturing of hydrate-bearing formations and their confining layers, with potentially adverse consequences on the stability of the suboceanic subsurface. This study also aims to determine whether the loss of the hydrate - known to have a strong cementing effect on the porous media - in the vicinity of the well, coupled with the significant pressure increases, can undermine the structural stability of the well assembly.

Scoping 1D simulations indicated that the formation intrinsic permeability, the pore compressibility, the temperature of the produced fluids and the initial hydrate saturation are the most important factors affecting the system response, while the thermal conductivity and porosity (above a certain level) appear to have a secondary effect. Large-scale simulations of realistic systems were also conducted, involving complex well designs and multilayered geologic media with non-uniform distribution of properties and initial hydrate saturations that are typical of those expected in natural oceanic systems. The results of the $2 \mathrm{D}$ study indicate that although the dissociation radius remains rather limited even after long-term production, low intrinsic permeability and/or high hydrate saturation can lead to the evolution of high pressures that can threaten the formation and its boundaries with fracturing. Although lower maximum pressures are observed in the absence of bottom confining layers and in deeper (and thus warmer and more pressurized) systems, the reduction is limited. Wellbore designs with gravel packs that allow gas venting and pressure relief result in substantially lower pressures.
\end{abstract}

\section{Introduction}

\section{Background.}

Gas hydrates are solid crystalline compounds in which gas molecules (referred to as guests) are lodged within the lattices of ice crystals (called hosts). Under suitable conditions of low temperature and high pressure, a gas G will react with water to form hydrates according to

$\mathrm{G}+N H \mathrm{H} 2 \mathrm{O}=\mathrm{G} \cdot N H \mathrm{H} 2 \mathrm{O}$,

where $N H$ is the hydration number. Of particular interest are hydrates formed by hydrocarbon gases when $\mathrm{G}$ is an alkane. Natural hydrates in geological systems also include CO2, H2S and N2 as guests. Vast amounts of hydrocarbons are trapped in hydrate deposits1. Such deposits occur in two distinctly different geologic settings where the necessary low temperatures and high pressures exist for their formation and stability: in the permafrost and in deep ocean sediments.

The three main methods of hydrate dissociation are 1: (1) depressurization, in which the pressure $P$ is lowered to a level lower than the hydration pressure $P e$ at the prevailing temperature $T,(2)$ thermal stimulation, in which $T$ is raised above the hydration temperature $T e$ at the prevailing $P$, and (3) the use of inhibitors (such as salts and alcohols), which causes a shift in the $P e$-Te equilibrium through competition with the hydrate for guest and host molecules. Dissociation results in the production of gas and water, with a commensurate reduction in the saturation of the solid hydrate phase.

Gas hydrates exist in many configurations below the sea floor, including massive (thick solid zones), continuous layers, nodular, and disseminated, each of which may affect the seafloor stability differently. The hydrates in all of these configurations may be part of the solid skeleton that supports overlying sediments, which ultimately support platforms and pipelines needed for production from conventional oil and gas resources, and from hydrate accumulations (when it becomes economically and technically viable).

\section{Objective and Problem Description.}

The main objective of this study is to evaluate the response of marine Hydrate- Bearing Sediments (hereafter referred to as HBS) to thermal loading. Such thermal loading occurs when heat from hot reservoir fluids (produced 
from deeper reservoirs) flows into the HBS through uninsulated pipes.

The resulting rise in temperature in the HBS can have serious consequences. Even before dissociation is attained, a rising temperature $T$ is expected to affect the mechanical strength of hydrate-bearing sediments - possibly severely, given the narrow temperature range between hydrate stability and dissociation. When the temperature $T$ reaches the hydrate equilibrium $T e$ temperature (see Figure 1) at the prevailing pressure $P$ (usually close to the hydrostatic pressure at the location), hydrate dissociation occurs by thermal stimulation. This leads to the rapid release of large amounts of gas, which can in turn result in the evolution of high pressures. This higher pressure can result in formation fracturing, with potentially serious consequences if the fracture plane crosses the confining (impermeable) top boundary of an underlying reservoir, thus allowing the escape of the reservoir fluids. It is also possible that the increased $P$, if sufficiently high, can have detrimental effects on the wellbore assembly, including cement fracturing and wellbore collapse.

Another problem that can be potentially caused by thermal loading is the deterioration of the structural stability of the geologic formation in the vicinity of the wellbore. Hydrates are very effective cementing agents 2 , and their dissociation can lead to significant geomechanical changes in the thermally affected region (including substantial subsidence). Unless accounted for, these changes can pose a hazard to the structural stability of the wellbore assembly. The reason for the concern is demonstrated in the photograph of Figure 2, which shows a dissociating core of a marine HBS. While the more isolated inner portion of the core (where hydrate still remains) appears "solid" and structurally strong, the medium in the outer annular space (where hydrate dissociation is in progress or has already occurred) has a fluid and very weak consistency because of the loss of the cementing hydrate and shows evidence of escaping gases (bubbling). Because of its consistency, the remaining watery mud is characterized as "soupy sediment". The impact of its evolution on the structural stability of marine HBS demands evaluation, especially in the case of compressible sediments such as muds and clays. Finally, during dissociation, the basal zone of the gas hydrate becomes underconsolidated and possibly over-pressurized because of the newly released gas3, leading to a zone of weakness akin to that indicated in Figure 2 (i.e., low shear strength, where failure could be triggered by gravitational loading or seismic disturbances), which can result in submarine landslides4,5.

In this study, we employ numerical simulation to determine the effects of thermal loading on the behavior of marine hydrate deposits (assumed to be pure CH4 hydrates). More specifically, we determine the evolution over a 30-year production period of important conditions and parameters in the oceanic subsurface, including $P, T$, phase saturations, porosity (as affected by $P$ and $T$ ), and salt concentration (as influenced by the release of fresh water from the dissociating hydrates). We also determine the magnitude and location of the maximum pressure evolving in the dissociating HBS, and we provide estimates of the rate of gas release and of the cumulative volume of released gas. These data can be used as inputs in standard geomechanical models to determine the geomechanical and structural response of the oceanic subsurface, and to evaluate the safety and adequacy of proposed well designs.

\section{The numerical simulation code.}

The numerical studies in this paper were conducted using the TOUGH-Fx/HYDRATE simulator6, which can model the non-isothermal hydration reaction, phase behavior and flow of fluids and heat under conditions typical of natural CH4-hydrate deposits in complex geologic media. It includes both an equilibrium and a kinetic model7,8 of hydrate formation and dissociation. The model accounts for heat and up to four mass components (i.e., water, $\mathrm{CH} 4$, hydrate, and water-soluble inhibitors such as salts or alcohols) that are partitioned among four possible phases: gas phase, liquid phase, ice phase, and hydrate phase. A total of 12 states (phase combinations) can be described by the code, which can handle any combination of hydrate dissociation mechanisms and can describe the phase changes and steep solution surfaces that are typical of hydrate problems.

Contextual analysis of simulation predictions. Note that the TOUGH-Fx/HYDRATE simulator6 is a mass and heat flow and transport code, and does not include a full geomechanical component. Thus, it cannot internally estimate stresses and strains resulting from $P$ and $T$ changes in response to HBS heating and hydrate dissociation. As such, it cannot compute the effect of the resulting changes in stress and strain on the HBS hydraulic, thermal and geomechanical properties system, and on the overall system behavior (e.g., deformation and subsidence). However, TOUGH-Fx/HYDRATE can predict the evolution of all the other important hydraulic and hermal properties and conditions discussed earlier, which can then serve as inputs in subsequent geomechanical studies.

\section{Scoping Studies}


Purpose. In this component of the study we analyze the response of a simplified, infinite-acting, 1-D radial (singlewell) HBS system to heating by warm fluids rising to the surface through uninsulated tubing at the center of the domain. The purpose is to determine the sensitivity of the system response to the parameters that are most relevant in HBS systems.

System description. A domain of uniform properties and initial conditions, and consisting of a single layer (with $\Delta z$ $=1 \mathrm{~m}$ thick) was considered (Table 1), thus allowing the estimation of the response per unit thickness of HBS. The domain radius $r \max =1000 \mathrm{~m}$ was subdivided into 275 cells of non-uniform $\Delta r$. Because of the need to accurately describe heat transfer within and in the vicinity of the wellbore assembly, and because heat conduction (which is expected to play an important role in this study) is a slow process, a very fine discretization was used near the center of the radial grid.

Well description. The design and material properties of the wellbore assembly, along with details of the system geometry, are given in Table 2. In the discretized domain used in the simulation, the first 23 radial elements (out of the total of 275) are used to describe the well system. This fine discretization provides a high-resolution description of the critically important heat flow through the inner tubing, the five steel casings (including the outer conductor), and the corresponding cement- and brine-filled annular spaces. Note that the same well design was used in the ensuing large-scale 2-D studies of HBS heating in realistic natural hydrate accumulations. The innermost radial gridblocks in the 1-D and 2-D studies correspond to the region in which hot fluids flow through the tubing (hydraulically impermeable in the horizontal direction), and are designated as constant-conditions boundary cells. In the reference case of the scoping studies, the inner boundary cell is maintained at a $T W F=90 \mathrm{oC}(=194 \mathrm{oF})$.

Sensitivity analysis. The properties of the reference case of the simplified 1-D problem in the scoping calculations are listed in Table 1. Note that, for reasons already discussed, this study does not aim to directly determine structural stability, but rather investigates the system sensitivity to conditions that can potentially undermine the structural stability of the HBS. We tested the HBS system sensitivity to the following parameters: (a) the formation permeability $k$, (b) the formation porosity $\varphi$, (c) the pore compressibility $\alpha$, (d) the initial hydrate saturation $S H$, (e) the temperature of the fluids $T W F$ flowing through the tubing, and (f) the thermal conductivities of the saturated and dry porous medium $(k \Theta R W$ and $k \Theta R D$, respectively).

HBS reference system behavior. The HBS system behavior in the reference case (Table 1) is described by Figures 3, 4 and 5, which show the temporal evolution of the spatial distributions (along $r$ ) of $P, T$, and $S H$ and $S G$, respectively, during a 30-year simulation period. The HBS system behavior when the values of hydraulic, thermal and operational parameters (identified in Figures 6 and 7) are modified is entirely analogous. An important observation from Figure 3 is that the maximum pressure in the domain Pmax is observed in a subdomain that coincides roughly with the zone of complete dissociation (ZCD) defined by $0<r<r d 0$, where $r d 0$ is the radius $r$ at which $S H=0$ (see Figure 4). Pmax does not occur at a single location but is practically uniform within the ZCD, and decreases slowly with time as the advancing dissociation front makes a progressively larger volume available for gas storage Figures 5a and 5b).

The two marked changes in the slope of the $T$ curves in Figure 4 correspond to the outer radius of the well assembly $r w$ and $r d 0$, respectively. Consistent with expectations, the thermally disturbed zone is limited in extent because of the constant TWF temperature, the decreasing (with time) thermal gradient at the well (as its vicinity becomes warmer), and the rapidly increasing volume (and, consequently, mass) as the radius of the affected zone expands. For the same reasons, $r d 0$ remains limited even after 30 years of continuous heating (Figure 5a). Similarly, the width of the zone of active dissociation (ZAD), defined as the zone $r d 0<r<r d$, increases with time (Figures $5 \mathrm{a}$ and $5 \mathrm{~b}$ ), but it also remains limited. Note that the outer radius $r d$ of the ZAD represents the edge of the dissociation front that occurs where $S G>S G O$ and $S H<S H O$ (Figures $5 \mathrm{a}$ and $5 \mathrm{~b}$ ).

Figures 6 and 7 quantify the system sensitivity by showing the observed maximum pressure deviation $\Delta P(=P \max -$ $P 0)$ in the domain to variations in the hydraulic and thermal properties discussed earlier. Each one of the graphs in Figures 6 and 7 (as well as the corresponding ones in Figures 8 and 9, which will be described next) represents a variation in the single parameter indicated in the legend; all other parameters remain as in the reference case (Table 2). Pmax is the maximum pressure occurring in the hydrate-bearing portion of the domain under investigation at a given time. The magnitude and the extent of the zone of Pmax occurrence are important parameters determining the 
upper limit of the geomechanical response of a HBS under thermal stress.

Figures 8 and 9 show the volumetric rate $Q V$ of hydrateoriginating $\mathrm{CH} 4$ release corresponding to Figures 6 and 7 , respectively. A direct correlation is expected between an increasing $Q V$ and a rise in Pmax. The $\mathrm{CH} 4$ release is induced by thermal stimulation caused by heat flow from the well fluids into the formation through the well assembly, and is responsible for the pressure rise in the domain. An important observation from Figures 6 through 9 is that the maximum levels of Pmax and $Q V$ are invariably observed early (at $\mathrm{t}<200$ days) during the 30-year heating process.

Note that the $Q V$ fluctuations in Figures 8 and 9 (as well as in the subsequent $Q V$ curves of Figure 18) are an inevitable feature of the dissociation process6. Hydrate dissociation leads to an increase in the rate of $\mathrm{CH} 4$ release, which results in a localized increase in pressure (caused by the evolving gas and the resistance to flow posed by the porous medium) and a drop in temperature (caused by the endothermic nature of the hydratie dissociation reaction). The increase in pressure and the temperature drop result in a subsequent reduction in $Q V$ until conduction and convection (of the advancing warm fluids) can raise the temperature and accelerate dissociation. The result of this sequence of events is the oscillatory appearance (of a roughly periodic nature) of the $Q V$ curve, which is also affected by grid discretization effects6.

Sensitivity to $\boldsymbol{k}$. Consistent with expectations, Pmax increases with a decreasing $k$ (Figure 6a) because of reduced pressure dissipation (caused by increasing impedance to flow). This occurs despite the decrease in the rate of $\mathrm{CH} 4$ release $Q V$ with a decreasing $k$ (Figure $8 \mathrm{a}$ ), caused by the reduction in the advection of warmer fluids (a far more efficient mechanism of heat transport than conduction, with conduction becoming increasingly dominant in less permeable systems) outward from the well assembly. Note that the Pmax for $k=10-16 \mathrm{~m} 2(0.1 \mathrm{mD})$ and $k=10-17$ $\mathrm{m} 2(0.01 \mathrm{mD})$ correspond to pressure increases of about $13 \%$ and $59 \%$, respectively, relative to the initial $P 0$, and may be sufficient to cause significant geomechanical changes to the formation (including permeability and porosity changes, displacement, and fracturing). The low initial intrinsic permeabilities considered in these cases are not uncommon in muddy oceanic sediments rich in clays.

Sensitivity to $\varphi$. Counter to expectations (which would associate a lower $\varphi$ with a limited volume for gas expansion, and, consequently, higher pressures), Pmax increases with an increasing $\varphi$ (Figure 6b). This is because more hydrate is available for dissociation and gas generation when the hydrate saturation is kept constant (at $S H=0.5$ in the reference case). This is evident in Figure 8b, which shows a decline in $Q V$ as $\varphi$ decreases. Note that the effect of $\varphi$ on Pmax is much less pronounced for $\varphi>0.30$.

Sensitivity to $\alpha$. Pmax increases with a decreasing $\alpha$ (Figure 6c) because of reduced pressure dissipation caused by increasing resistance to deformation of the porous medium. The effect is non-linear, with Pmax increasing by only about $12 \%$ when $\alpha$ decreases by an order of magnitude. Note that in this case $Q V$ is relatively unaffected because $\mathrm{CH} 4$ is released at about the same rate for both values of $\alpha$ (Figure $8 \mathrm{c}$ ).

Sensitivity to SH. Pmax increases with an increasing SH (Figure 7a) because of the corresponding reduction in effective permeability, in a manner analogous to the effect of a decreasing $k$ discussed earlier. In the rate of $\mathrm{CH} 4$ release $Q v$ in Figure 9a, there are two processes in competition: the availability of hydrate (with higher $S H$ tending to lead to larger $Q v$ ), and the reduced relative permeability of fluids (which would tend to reduce advective transport of warmer fluids outward from the well, thus leading to lower $Q V$ ). It is evident from Figure 7a that the reduction in permeability outweighs the impact of the increased hydrate availability in the case of $S H=0.75$. The two processes are roughly in balance for $S H=0.25$ and $S H=0.50$. Despite the lower $Q V$ for $S H=0.75$, the reduced permeability results in a significant increase of Pmax compared to that for the reference case.

Sensitivity to $\boldsymbol{T W F}$. An increasing temperature of the rising reservoir fluids in the tubing is expected to lead to higher heat fluxes into the HBS, and consequently, larger $Q V$ and higher Pmax. The results in Figures $7 \mathrm{~b}$ and $9 \mathrm{~b}$ are consistent with these expectations, and show a nearly linear relationship between $Q V$ and $\Delta P$.

Sensitivity to $k \Theta R W$ and $k \Theta R D$. A reduction in the values of the thermal conductivities of the saturated and dry 
porous medium ( $k \Theta R W$ and $k \Theta R D$, respectively) leads to values of $Q V$ that are nearly equal to those for the reference case at early times, and are slightly lower at later times (Figure 9c). This is attributed to the slow nature of heat transfer through conduction, which results in relatively longer times before this process affects the system response. At early times, the effect of the lower thermal conductivities of the porous media are balanced by the resulting steeper temperature gradients, yielding approximately the same heat fluxes and resulting in very similar $Q V$. The effect of lower $k \Theta R W$ and $k \Theta R D$ on Pmax (Figure 7c) appears to be closely correlated with that on $Q V$ (Figure 9c), with lower thermal conductivities leading to practically the same Pmax at earlier times (when the highest levels are observed), and slightly lower Pmax after prolonged heating, relative to the reference case.

\section{Analysis of Realistic 2D Marine Hydrate Systems}

Geologic system description. Although the large-scale 2D problem and its three variations discussed in this section are synthetic, they involve realistic representations of the geology and conditions occurring in natural oceanic HBS. In all cases, the geological system of interest was composed of a layered sequence of five formations (A through E), confined at the top and bottom by impermeable (or nearly-impermeable) shale layers (designated as formations $U$ and $\mathrm{L}$, respectively). The five formations had the same hydraulic properties and initial fluid saturation distributions in all the test cases of this study (see Table 3). All other properties and parameters remained as in Table 1 of the scoping study.

The thickness of the shale in the upper boundary was $50 \mathrm{~m}$. There was a downward trend of the intrinsic permeability $k$ with depth, declining from $10-15 \mathrm{~m} 2(1 \mathrm{mD})$ in the shallower formation A to $5 \times 10-18 \mathrm{~m} 2(0.005 \mathrm{mD})$ in the deepest formation E. Similarly, the porosity $\varphi$ decreased from 0.50 in formation A to 0.40 in formation E. These variations are consistent with the increased compaction of marine formations with depth. The relatively large $\varphi$ values (generally higher than those observed in onshore reservoirs) are often observed in unconsolidated oceanic sediments 11. Conversely, the initial hydrate saturation $S H$ generally increased with depth from 0.15 in formation A to 0.50 in formation E, and were within the reported range in similar systems. These variations in $k$, $\varphi$ and $S H$ with depth were consistent with field observations.

The cases investigated in this $2 \mathrm{D}$ study are the following:

Case A: Reference case. The reference case for this problem involves the system and conditions described in Tables 1 and 3. Heat from the hot fluids in the inner tubing flows through the well assembly to the hydrate-bearing formations, causing a temperature rise and resulting in hydrate dissociation. The confining formations (top and bottom boundaries) are impermeable, but allow heat exchange with the overburden and the underburden. Consequently, the gas released from dissociation cannot escape into the ocean, but remains trapped in the system and leads (inevitably) to higher pressures. The geothermal gradient in this case (as well as in cases C and D, see below) to the base of the hydrate (i.e., at the bottom of layer E) was $d T / d z=0.0332 \mathrm{oC} / \mathrm{m}$. With this geothermal gradient, the base of the hydrate coincided with the bottom of the hydrate stability zone at the $P=12.4 \mathrm{MPa}$ at that elevation.

Case B: Deeper deposit and different geothermal gradient. As can be seen in Table 3, case B involved a deeper mudline (at an elevation of $-1500 \mathrm{~m}$, as compared to $-1000 \mathrm{~m}$ for all other cases). With pressures in the HBS following the hydrostatic gradient, this resulted in a higher-pressure system. The temperature at the mudline was kept at $5 \mathrm{oC}$. In case $\mathrm{B}$, the geothermal gradient $d T / d z=0.0446 \mathrm{oC} / \mathrm{m}$, resulting in (a) hydrate occurrence throughout the 5-layer (A to E) profile and (b) the bottom of Formation E coinciding with the bottom of the hydrate stability zone, at which $P=17.33 \mathrm{MPa}$. The steeper geothermal gradient results in higher temperatures in the upper parts of the profile, leading to expectations of higher rates of gas generation from hydrate dissociation and higher pressures. The reason for the consideration of this case was the evaluation of the relative performance of a deeper (and, consequently, warmer and more pressurized) system exposed to thermal stresses.

Case C: Absence of bottom confining shale layer. The geological system in Case $\mathrm{C}$ differs from the reference Case A in that there is no bottom shale layer (impermeable formation L, see Table 3) coinciding with the bottom of the hydrate stability zone. Thus, the shale layer is now replaced by a deep hydrate-free layer with the properties of Formation E and $300 \mathrm{~m}$ thick. Using the terminology of Moridis and Collett 9 , this configuration describes a Class 2 hydrate deposit, with all other cases representing Class 3 hydrate accumulations. The absence of the lower confining 
impermeable layer is expected to result in lower pressures because the released gas can expand into a larger permeable volume (compared to the reference case, in which hydrate-originating gas can only move through the HBS away from the well towards the outer boundaries). The reason for the consideration of Case $\mathrm{C}$ is the assessment of the importance of such boundaries to the system response.

Case D: Pressure release. The only difference between Case D and the reference Case A is the inclusion of highpermeability region $(k=10-11 \mathrm{~m} 2=10000 \mathrm{mD})$ that extended radially $0.1 \mathrm{~m}$ into the formation from the outermost cement "ring" of the well assembly. The high $k$ in this region is attributed to a sand or gravel pack emplaced during drilling and well construction operations, which are also assumed to have caused dissociation of the hydrate originally located there. The high-permeability region extends to from the bottom of formation $\mathrm{E}$ to the mudline, at which point creates a constant pressure boundary (equal to the hydrostatic pressure at the bottom of the ocean).

This configuration is designed to provide pressure relief by allowing gas released from hydrate dissociation to escape into the ocean, and is expected to result in the evolution of lower pressures in the HBS. Such lower pressures are desired to prevent wellbore collapse, and/or to avoid the inadvertent fracturing of the hydrate or of the shale overburden and underurden. The reason for the consideration of Case $\mathrm{D}$ is the evaluation of a well design to mitigate the potentially adverse effects of higher pressure.

\section{Simulation specifics}

Domain discretization and boundary conditions. An infinite-acting single-well system (described by a cylindrical domain) was used in the simulations. The outer reservoir radius was placed at rmax $=1000 \mathrm{~m}$, where an outer constant conditions boundary was located. Based on scoping calculations, the domain included only $30 \mathrm{~m}$ of the bottom-confining layer (i.e., the no-flow but heat-exchanging lower shale boundary) when underburden was considered (Cases A, B and D). The 30-m thickness is sufficient to allow accurate heat exchange with the hydrate deposit during the 30 -yr long production period. The thicknesses of the five hydrate-bearing formations (A to E) are described in Table 3.

The 2-D cylindrical domain was discretized in 194 x $100=19,400$ gridblocks in $(r, z)$. The uppermost and lowermost layers corresponded to constant temperature, no-flow boundaries, while the layers corresponding to the top and bottom confining layers (formations $\mathrm{U}$ and $\mathrm{L}$, see Table 3) were impermeable but allowed heat exchange between the deposit and its surroundings. To describe accurately the processes and phenomena occurring in the critically important vicinity of the wellbore, and because the extent of the ZCD and ZAC are expected to be limited (see Figures 3 to 5), we used a very fine discretization in the HBS along the $r$ direction (especially in the $r<20 \mathrm{~m}$ zone). Using the equilibrium hydration reaction option in TOUGH-Fx/HYDRATE, the grid resulted in 77,600 coupled equations that were solved simultaneously.

Initial conditions. We determined the initial conditions in the reservoir by following the initialization process described by Moridis et al.10. The initial temperature distribution was computed from the known temperature at the mud line (reported as $5 \mathrm{oC}$ ) and the corresponding geothermal gradients. The pressures in the subsurface profile were assumed to follow a hydrostatic distribution. This is consistent with observations of natural hydrate accumulations in geologic media12. The initial pressures were computed using the water depths reported earlier, and a pressure-adjusted saline water density typical of ocean water $(1003 \mathrm{~kg} / \mathrm{m} 3$ at atmospheric pressure).

Well description. The well design is the same as the one described earlier in the scoping study (see Table 2). In the discretized domain used in the 2-D simulation, the first 23 radial elements (out of a total of 194 at each elevation) are used to describe the well system (to $r=r w$ ), thus providing the high resolution needed to capture the heat transfer within the well assembly. In all cases, the temperature of the rising hot fluids in the tubing was $T W F=90$ $\mathrm{oC}(=194 \mathrm{oF})$.

Simulation process and outputs. The simulation period was 30 years, corresponding to the typical production life span of the deeper hot reservoir. Because the well assembly is radially impermeable, only heat is exchanged between the hot production fluids and the colder (initially) hydrate-bearing formations. As a result, $T$ in the HBS increases, 
hydrate dissociation begins, gas and water are released, and the pressure rises. Dissociation is a self-limiting reaction because it is strongly endothermic (thus consuming a large portion of the heat supplied by the wellbore), and because the rising pressure makes further dissociation progressively more difficult.

In the course of the simulation, the following conditions and parameters are monitored: Spatial distributions (at $t=1$ year, 5 years, 10 years, 20 years and 30 years of (a) pressure, (b) temperature, (c) aqueous, gas and hydrate phase saturations, (d) water salinity (as affected by the evolving pressure and temperature), and (e) porosity; Location and magnitude of maximum pressure; Volumetric rate of $\mathrm{CH} 4$ released from dissociation from the entire hydrate-bearing system $(Q V)$; and Cumulative volume of $\mathrm{CH} 4$ released from dissociation $(V C)$. In Case $\mathrm{D}$, the following variables are also monitored: volumetric rate of gas produced from the annular gravel pack $Q P$, mass water production rate from the gravel pack $Q W$, and cumulative gas volume and water mass produced from the gravel pack ( $V C$ and $M W$, respectively) during the 30 -year period.

\section{D System Response to Heating in Case A}

Despite their differences, the system response was consistent in pattern and similar in magnitude in all four cases. This allows us to discuss the spectrum of the results by using a basic set as an example of the general pattern of response, while using focused datasets (and the corresponding figures) to discuss differences in the system behavior caused by a variety of factors. In this section, we use the evolution of the spatial distributions of important variables in Case A (i.e., the reference case) as the basis of discussion of the system behavior.

Spatial distributions of $\boldsymbol{P}$. The $P$ distribution in the reference case in Figure 10 indicates that the region of maximum pressure Pmax is observed in the lowermost hydrate-bearing formations (where the lowest $k$ and $\varphi$, and the highest $S H$ occur). A very important observation from Figure 10 is that the pressure rise is very significant (about $9 \mathrm{MPa}$, i.e., a $70 \%$ increase of $P 0$ at the bottom of formation E), but the high-pressure region is confined to a small volume in the vicinity of the well and does not appear to extend significantly past $r=25-30 \mathrm{~m}$ (in a domain with an $\operatorname{rmax}=1000 \mathrm{~m}$ ) even after 30 years of continued heating. The high-pressure region is restricted to formations $\mathrm{C}$ through $\mathrm{E}$ (with pressure increasing with depth), and does not appear to extend to the upper formations $\mathrm{A}$ and $\mathrm{B}$. This is because of the relatively high permeabilities and low initial hydrate saturations in formations A and B. It is evident that the bulk of significant geomechanical changes will occur in the high-pressure region. Despite their limited extent, it is reasonable to expect that the significant pressure changes we observe can have adverse effects in weak marine sediments. Because no information is currently available on the geomechanical behavior of HBS, it is not possible to predict whether these pressure changes are sufficient to effect fracturing of the HBS or of the continuing shale layers.

Spatial distributions of $\boldsymbol{T}$. The $T$ distribution in Figure 11 indicates that the temperature rise caused by the heating from the wellbore affects a narrow cylindrical zone, as is depicted by the very faint color variation over time within the $r=25 \mathrm{~m}$ zone of observation. In order to highlight the temperature distribution beyond $r=2 \mathrm{~m}$, the color scale is saturated at $30 \mathrm{oC}$. Note that, unlike the scoping studies (Figures 3 and 4), neither the $P$ distributions in Figure 10 nor the $T$ distributions in Figure 11 can provide a satisfactory approximation of the location of the hydrate dissociation front (see Figure 12). If the relationship of a rising $T$ on the mechanical strength of HBS is known, comparison of the $T$ distributions in Figure 11 and the SH distributions in Figure 12 can provide an estimate of the extent of the region that is structurally compromised (and subject to deformation and subsidence) by weakened HBS.

Spatial distributions of $\mathbf{S H}$. The most important observation from the review of the hydrate saturation distribution in the reference case in Figure 12 is the limited extent of the hydrate dissociation zone, which decreases with depth and is barely larger than $r d 0=15 \mathrm{~m}$ at its maximum after 30 years of heating. The $r d 0$ ranges from $15 \mathrm{~m}$ in the uppermost formation $\mathrm{A}$, to $10 \mathrm{~m}$ in the lowermost formation $\mathrm{E}$, and is very small compared to the Rmax $=1000 \mathrm{~m}$ of the simulated domain. The outer radius $r d$ of the ZAD is also very small, decreasing with depth from about $32 \mathrm{~m}$ in formation $\mathrm{A}$ to $14 \mathrm{~m}$ in formation $\mathrm{E}$.

The limited $r d 0$ and $r d$ were consistent with expectations because the cylindrical configuration of the system results in rapidly increasing volumes (as the radius increases) supplied with heat by a constant-temperature boundary (the inner tubing). Thus, a progressively decreasing fraction of the released heat reaches the dissociation front, the rest being consumed to maintain the temperature of the increasing volume of the hydrate- free (after dissociation) 
cylindrical zone around the well. Additionally, the heat flux from the constant-temperature boundary continuously decreases because of the increasing formation temperature that leads to smaller temperature differentials, thus weakening the driving force of heat transfer.

Note the creation of a thin zone of "new" hydrate in the uppermost region of layer A, where the released gas accumulates (because of buoyancy) and where $T$ is relatively low. Consistent with the scoping studies (Figure 5a), formation of new hydrates is also observed ahead of the dissociation fronts in the various layers (as indicated by the slightly darker color at these locations, e.g., layers B and C).

The staircase appearance of $S H$ distributions in Figure 12 is attributed to the layered pattern (Table 3 ) of decreasing $k$ and increasing $S H$ with depth ( $\varphi$ has been shown to be insensitive in this range, see Figure $6 \mathrm{~b}$ ). While the larger $\mathrm{SH}$ results in a lower composite heat capacity of the HBS (and, thus, in a faster response to heating), the effect is outweighed by the reduction in the relative permeability of fluids. Consequently, the advection of warm fluids (an effective mechanism of heat transfer) moving radially outward from the well is increasingly restricted with depth, while the contribution of the far more inefficient conduction increases. This results in heat transport, hydrate dissociation and ZAD that increase with the effective permeability of the system, hence the staircase $S H$ pattern.

An additional reason for $r d 0$ and $r d$ decreasing with depth is the larger pressure that results from the gas release in the lower formations (Figure 10), which acts as an impediment to dissociation. Conversely, the higher permeability and lower hydrate saturations in the upper formations allow gas flow toward the outer radius of the reservoir, prevent pressure build-up, and result easier dissociation.

While the ZCD and ZAD may be limited, significant concerns regarding the geomechanical behavior of this system need to be alleviated if wells are to be installed under such conditions. The question that begs to be answered is whether the ZCD (at the center of which lies the well) can provide stability by supporting the considerable weight of the wellbore assembly if a weak unconsolidated porous medium is involving, leaving "soupy sediments" (Figure 2) after the dissociation of the hydrate. The issue becomes more critical if the production system design involves close spacing of multiple wells. Lack of fundamental knowledge precludes any reliable predictions at this time.

Spatial distributions of $\boldsymbol{S} \boldsymbol{G}$ and $\boldsymbol{S} \boldsymbol{A}$. The $S G$ and $S A$ phase saturation distributions in the reference case (Figures 13 and 14, respectively) roughly mirror the hydrate saturation distribution in Figure 12. Note that the occurrence of high $S G$ in the ZCD near the wellbore can have a negative impact on the structural stability of that region because of the very high compressibility of gases.

Of interest is the drainage of water (originating from the dissociating hydrate) observed in Figure 14, which is particularly evident in the upper formations. The drainage of water is also reflected in the $S G$ distribution in layer A of Figure 13, where it is depicted as a reduction in the gas saturation.

A feature common to all phases (Figures 12 to 14) is the wedge-like appearance of the saturations at the bottom of each formation, where the dissociation front intrudes deeper into the hydrate layer. This is caused by the preferential upward flow patterns of hydrate-originated fluids released from the underlying less permeable formations. This leads to enhanced dissociation in the upper

Spatial distribution of porosity changes $\Delta \varphi=\varphi-\varphi \boldsymbol{0}$. As expected, the porosity deviations (from their initial levels) in the reference case in Figure 15 follow closely the pressure distributions in Figure 12, and are limited in areal extent. An increasing pressure leads to an increasing porosity, and the change appears to be substantial in this study. Note that these results were not obtained using a geomechanical model, but were predicted by the TOUGHFx/HYDRATE6 simulator that accounts for the $P$ and $T$ effects on $\varphi$ only (and not on permeability) using reasonable (but assumed and unverified) pore compressibility and expansivity estimates. As such, these results should be considered as indicative only. Note that if the pore changes shown in Figure 15 are correct, significant sediment displacement (although localized) is possible, the effects of which on the system structural stability is unknown because of lack of data.

Spatial distribution of salinity. The salinity distribution may have a significant effect on the shale boundaries because these would tend to swell and lose their structural integrity when exposed to fresh water released from hydrate dissociation. As expected, Figure 16 shows pervasive low-salinity water regions that roughly coincide with the ZCD, closely following the staircase patterns and extent of the phase saturation distributions (Figures 12 to 14). The low salinity is caused by the mixing of the native saline water with the fresh water released from hydrates 
during dissociation. The region of minimum salinity is shown to coincide with the deepest HBS in layer E, and is consistent with expectations as the location of accumulation of the fresh water draining from higher elevations.

Additionally, the locations of formation of new hydrate are clearly depicted in Figure 16 as regions of elevated salinity, and help to identify the upper boundaries of the ZAD. These are observed in the uppermost reaches of layer $\mathrm{A}$, and as wedges at the bottom of each of the formations following the advancing gas front along the bottom boundary of the system. An area of elevated salinity is also observed, coinciding with the location of hydrate formation. There is currently no quantitative information on the mechanical properties of swelling marine shales to evaluate whether the predicted salinity distribution will be a cause for concern during similar production operations.

\section{Sensitivity Analysis of the 2D System}

Evolution of maximum pressure. The maximum pressure attained within the hydrate-bearing layers of the simulated system, as well as the corresponding location, are of critical importance because they determine the potential for fracturing the HBS and/or the confining impermeable layers, in addition to providing a measure of the damage hazard to the wellbore assembly. Based on anecdotal evidence, because of the unconsolidated, unlithified nature of such waterlogged media and their very high porosity (see Figure 2), the fracture pressure of the formations can be quite low and not significantly larger than the initial pressure.

Figure 17 shows the evolution of maximum pressure in Cases $\mathrm{A}$ through $\mathrm{D}$. Review of the maximum pressure evolution in Case $\mathrm{A}$ indicates that the maximum pressure increase $\Delta P=P \max -P 0$ increases rapidly during the early stages of thermal loading (starting at $t<1$ day), rises to $\Delta P=8.0 \mathrm{MPa}$ in about 30 days, and reaches a maximum level of $8.8 \mathrm{MPa}$ (i.e., about $70 \%$ higher than the initial pressure) after about 280 days, after which time it begins to decline very slowly (dropping to $\Delta P=7.6 \mathrm{MPa}$ at $\mathrm{t}=30$ years). This indicates that, after a short initial period (during which heat from the wellbore reaches a rapidly expanding zone and the released gas causes the pressure to rise fast), the maximum pressure remains practically stable as the hydrate dissociation front (i.e., the locus of Pmax) advances. This is attributed to (a) the slowing rate of advance of the dissociation front (because the cylindrical volume of the thermally affected zone increases fast as a function of the square of the radius), and (b) an approximate balance between the rate of heat addition (originating from the hot fluids flowing in the inner tubing) through the wellbore assembly and the rate of heat consumption needed to sustain the strongly endothermic hydrate dissociation reaction.

The evolution of $\Delta P$ in Cases $\mathrm{B}$ and $\mathrm{C}$ is remarkably similar in pattern to that in Case $\mathrm{A}$. The onset of the rapid Pmax rise practically coincides in the isolated domains of Cases A, B and C. However, counter to expectations, Figure 17 indicates that the absence of a lower impermeable boundary (Case C) does not have a significant moderating effect on $\Delta P$, the maximum level of which is lowered only slightly to $\Delta P=8.1 \mathrm{MPa}$ (observed at about $t$ $=100$ days). Additionally, the $\Delta P$ in Cases $\mathrm{A}$ and $\mathrm{C}$ coincide until about $\mathrm{t}=10$ days (when over $90 \%$ of the maximum $\Delta P$ has already been attained), after which time the absence of the lower confining boundary is reflected by the lower $\Delta P$.

The evolution of $\Delta P$ in the case of the deeper system of Case $\mathrm{B}$ was also unexpected. The anticipation was that the higher temperature of the deeper formation would lead to a larger $\max \{\Delta P\}$, given the decrease in the heat of dissociation at $\mathrm{n}$ elevated $T$. However, $\Delta P$ in Case $\mathrm{B}$ is consistently lower than in Case $\mathrm{A}$, and reaches a $\max \{\Delta P\}=$ 7.7 MPa at about $t=300$ days (corresponding to about a $45 \%$ increase over $P 0$ ). Given the fact that $P 0$ is significantly higher in Case $\mathrm{B}(=17.33 \mathrm{MPa}$ at the base of formation $\mathrm{E}$, as opposed to $12.40 \mathrm{MPa}$ at the same location in Case A), it appears that the impact of thermal loading is relatively less pronounced in deeper formations. This is attributed to increased gas solubility at the higher pressures of the deeper formation of Case B, which outweighs the relatively mild (and opposite) effect of elevated T on CH4 solubility.

$\Delta P$ increase in Case $\mathrm{D}$ (in which pressure relief is provided through gas venting) is consistently and significantly lower than in all other case. This was expected because of the high-permeability zone in the annular gravel pack and the constant- pressure boundary, through which gas evolving from the hydrate dissociation can escape into the ocean (thus preventing a pressure build-up). The $\max \{\Delta P\}=6.0 \mathrm{MPa}$ (corresponding to about a $48 \%$ increase over $P 0$ ) is reached much later than in all other cases at about $t=1500$ days. While the Pmax observed in Case D is substantially lower than in all other cases, it is unknown (given the lack of information on the mechanical behavior of HBS) if it is at a level that can be considered safe for the prevention of formation fracturing at that location.

Review of the locations of Pmax during the simulation period indicates that these are always confined in the lowermost regions of the system (the bottom gridblock layers corresponding to the hydrate-bearing formation E). This was expected because at these locations (a) the initial temperature is higher because of the deeper formation (thus allowing easier dissociation after heating by the wellbore), (b) the permeability is lower, while (c) the hydrate saturation is higher, as is the composite thermal conductivity (the main heat transfer mechanism from the wellbore). 
Consequently, the large amounts of available hydrates can dissociate easily and fast, while the hydrateoriginating gas is released into a smaller "free" pore volume and cannot flow easily away from the dissociation front. Note that Pmax occurs within the bottom $10 \mathrm{~m}$ in cases A and B, while its range of occurrence extends to the bottom $23 \mathrm{~m}$ in Case $\mathrm{C}$, and narrows considerably to the bottom $5 \mathrm{~m}$ in Case $\mathrm{D}$.

Evolution of volumetric rates of gas release. Figure 18 shows the evolution of the volumetric rate $Q V$ of gas released from hydrates in the entire simulated domain in Cases A to $\mathrm{D}$. The $Q V$ increases rapidly in all cases during the early phase of dissociation (to $t=10$ days). Because of the higher $T$ and the corresponding lower heat of dissociation, $Q V$ is higher in case B than in the reference case. The absence of a confining structure next to the site of early dissociation in Case C leads initially to a lower (than in case A) $Q V$. A lower initial $Q V$ is also observed in Case $\mathrm{D}$, caused by the assumption of a hydratefree annular gravel pack at the location of early dissociation.

The evolution of $Q V$ in Case $\mathrm{B}$ is of particular interest.

Figure 18 indicates a distinct tendency for a large gas release, which becomes apparent at about $t=40$ days, culminates in a very rapid gas release starting at about 500 days, is followed by a period of hydrate reformation and re-dissociation in the $1200<t<3000$ period, and concludes with a gradual and consistent $Q V$ decrease for $t>3000$ days. Repeating the simulation with different grids (first half as coarse, and then twice as fine as the one used in these simulations) produced the same results, indicating that this process is not a numerical artifact related to grid discretization. Our current hypothesis is that the deeper (and warmer) formation is more sensitive to external thermal stresses than shallower colder ones, leading to violent phase transitions and reversals in response to small changes in $P$ and $T$. This sensitivity appears to occur very close to hydration equilibrium and to be related almost exclusively to phase composition. The fact that the corresponding $\Delta P$ in Cases B (see Figure 17) appears relatively smooth without any of the violent swings that $Q V$ exhibits lends credence to this hypothesis.

A comparison between the $\Delta P$ and $Q V$ in Cases A and B (Figures 17 and 18, respectively) indicates that although $Q V$ in Case B is larger than in Case A, $\Delta P$ is lower. This observation provides further support to the thesis that increase gas dissolution is the reason for the lower Pmax.

The absence of a confining structure in Case $\mathrm{C}$ allows the flow of warm fluids into the hydrate-free formation underneath layer E, thus depriving the HBS of their dissociation fueling heat. This leads to a reduced overall dissociation and a lower $Q V$.

Finally, the $Q V$ in case $\mathrm{D}$ is consistently higher than in the reference case because the continuous pressure relief lowers the pressure in the HBS and enhances dissociation. It is obvious that loss of warm fluids into the ocean through the highly permeable gravel pack is not sufficient to counter the effect of venting. The reason for the lower $P$ max in Case C (see Figure 17) despite the larger $Q V$ is demonstrated in Figures 19 and 20, which show the (a) volumetric rate $Q P$ of gas production (venting) and (b) the mass flow rate of water production into the ocean through the gravel pack, respectively. Figures 19 and 20 show that in addition to considerable amounts of water, about $20-30 \%$ of the gas released from hydrate dissociation escapes into the ocean, thus alleviating pressure buildup.

No gas is vented in the first 12 days (Figure 19). During this early period, dissociation begins, the pressure in the system gradually increases, and the hydrate-originating gas establishes a mobile gas phase and slowly rises through the gravel pack toward the ocean floor. During the same period, water is expelled at increasingly higher rates $Q W$ (Figure 20), which reach a maximum when a continuous gas phase is established in the gravel pack from the bottom of the system to the ocean floor and the first gas is vented into the ocean (Figure 19). The sudden pressure release leads to a significant pressure lowering, which allows water to rush back into the gravel pack and reduce $Q P$. Continuous gas release from dissociation rebuilds the pressure, a second release follows, and the process is repeated (leading to the oscillations in figures 19 and 20) until the gas phase is continuous and fully established. When the two-phase flow regime is stable in the gravel pack, the water flow rate $Q W$ (Figure 20) drops to a significantly lower level and exhibits a very slow decline during the 30 -year simulation period. Conversely, $Q P$ shows no similar drop upon the establishment of a steady flow regime, but is characterized instead by a continuous decline.

Evolution of cumulative volumes rates of released gas. The cumulative volumes $V C$ of $\mathrm{CH} 4$ released from hydrate dissociation (corresponding to the $Q V$ in Figure 18) appear in Figure 21. The log-log scale of Figure 21 makes the $V C$ appear similar in all cases, although slight differences are evident: with the exception of very early times $(t<10$ days), the relative size of the $V C$ is as follows: Case $\mathrm{D}>$ Case $\mathrm{B}>$ Case $\mathrm{A}>$ Case $\mathrm{C}$. This holds true until about $t=1000$ days, when the sudden gas release in the deeper and warmer system of Case B (Figure 18) leads 
to a quantum (step) increase in the corresponding $V C$.

The cumulative volumes of gas $V P$ and the cumulative

mass of water $M W$ that escape into the ocean are shown in Figures 19 and 20, respectively. Comparison of $V P$ to the $V C$ in Figure 21 shows that, at the end of the 30-year production period, $106 \mathrm{~m} 3$ of $\mathrm{CH} 4$ will have been vented into the ocean. This very significant volume represents about $30 \%$ of the total gas released from hydrate dissociation, and is sufficiently large to require appropriate actions for the collection or disposal of the released gas.

\section{Conclusions}

(1) During heating of an HBS by warm reservoir fluids rising through a well, the highest pressure Pmax that develops in the domain is practically uniform in the zone between the well radius $r w$ and the radius $r d 0$ of the zone of complete dissociation (ZCD). The thermally disturbed zone is limited even after prolonged heating, as is the dissociation radius. The extent of the zone of active dissociation (ZAD, $r d 0<r<r d$ ) increases with time, but this also remains limited.

(2) During heating of an HBS by warm reservoir fluids rising through a well, Pmax increases with (a) a decreasing $k$ and $\alpha$, and (b) with an increasing $S H, T W F$, and $\varphi$ (below a certain level). The sensitivity of Pmax to $k \Theta R W$ and $k \Theta R D$ is limited, and becomes significant only at later times.

(3) In addition to studying a reference case involving a large realistic hydrate-bearing system in the oceanic subsurface, three variations of the problem considered (a) the influence of depth and of the geothermal gradient, (b) the effect of boundaries, and (c) well designs and configurations for pressure release. In all four cases, the response to thermal loading (caused by warm reservoir fluids rising to the surface through unisulated pipes that cross the HBS) is similar in pattern, though not in magnitude.

(4) In all cases, the maximum pressure Pmax occurs consistently at the deepest hydrate-bearing layers. In enclosed systems with no pressure release (Cases A, B and C), Pmax increases rapidly during the early stages of thermal loading (starting at $t=1$ day), rises to a maximum within 100-300 days, and then decreases consistently (but very slowly) over the remainder of the 30-year simulation period. In case D (involving pressure release to the ocean through an annular gravel pack), the maximum Pmax was much lower, and occurred much later (i.e., at $\mathrm{t}=1000$ days).

(5) The maximum pressure changes $\Delta P=P \max -P 0$ represented increases between $45 \%$ and over $70 \%$ over the initial $P 0$. Without any information on the geomechanical properties and behavior of HBS, it is unknown if such pressures are above the safety threshold for formation fracturing and wellbore collapse.

(6) Compared to the reference Case A, $\Delta P$ in Case B is lower (despite higher rates $Q V$ of gas release from hydrate dissociation) because of increased solubility at the higher pressures of the deeper system.

(7) $\Delta P$ in Case $\mathrm{C}$ is slightly lower than in the reference Case $\mathrm{A}$ (and higher than in Case $\mathrm{B}$ ), because of lower rates $Q V$ of gas release from hydrate dissociation (as warm fluids into the hydrate-free formation underneath the HBS, thus depriving the hydrates of their dissociation-fueling heat). Thus, the absence of a lower impermeable boundary does not appear to have a significant moderating effect on $\Delta P$.

(8) The lowest $\Delta P$ is observed in Case $\mathrm{D}$. This is caused by the continuous escape of gas and water into the ocean through the annular gravel pack, which is sufficient large to negate the effects of the larger $Q V$ in this case. Thus, about $30 \%$ of the gas released from dissociation is vented into the ocean. The magnitude of the vented volume (106 $\mathrm{m} 3$ over 30 years) is sufficiently large to require collection and commercial exploitation (if feasible) or safe disposal to avoid environmental degradation.

(9) In the large 2D systems, the high-pressure region (developed in response to gas release from hydrate dissociation) increases with depth, is confined to a small volume in the vicinity of the well (not extending significantly past $r=30 \mathrm{~m}$ after 30 years of continuing heating), and is restricted to the lowermost formation.

(10) The temperature rise (caused by heating from the wellbore) is limited to a very narrow cylindrical zone, which is not a good indicator of the ZCD or ZAC of the HBS.

(11) The extent of the hydrate dissociation zone is limited, and does not extend past $r=10-15 \mathrm{~m}$ at its widest point after 30 years of continuous heating. The radii of the ZCD and ZAD increase with an increasing $k$ and a decreasing $S H$. It is not known if the formation is weakened to the point of being unable to support the considerable weight of the wellbore assembly after the loss of the cementing hydrate through dissociation. 
(12) The $S G$ and $S A$ distributions of mirror the $S G$ distributions over time. The occurrence of a high $S G$ in the ZCD can adversely affect the wellbore stability after the hydrate dissociation if soft, compressible and unconsolidated porous media are involved.

(13) The salinity distribution may have a significant effect on the shale boundaries because these would tend to swell and lose their structural integrity when exposed to fresh water released from hydrate dissociation.

\section{Nomenclature}

$\Delta r=$ Radial increment $(\mathrm{m})$

$\Delta t=$ Timestep size (s)

$\Delta z=$ Vertical discretization, i.e., in the z-direction (m)

$C=$ specific heat $(\mathrm{J} / \mathrm{kg} / \mathrm{K})$

$k=$ intrinsic permeability $(\mathrm{m} 2)$

$k \Theta=$ thermal conductivity $(\mathrm{W} / \mathrm{m} / \mathrm{K})$

$k \Theta R D=$ thermal conductivity of dry porous medium

$(\mathrm{W} / \mathrm{m} / \mathrm{K})$

$k \Theta R W=$ thermal conductivity of fully saturated porous

medium $(\mathrm{W} / \mathrm{m} / \mathrm{K})$

$M W=$ cumulative mass of water released into the ocean

through the annular gravel pack $(\mathrm{kg})$

$N H=$ hydration number

$P=$ pressure $(\mathrm{Pa})$

$P 0=$ initial pressure in hydrate-bearing sediments $(\mathrm{Pa})$

$P \max =$ maximum pressure in the domain at any given time

$(\mathrm{Pa})$

$\Delta P=P \max -P 0(\mathrm{~Pa})$

$Q P=$ volumetric rate of gas release into the ocean

through the annular gravel pack (ST m3/s)

$Q W=$ mass rate of water release into the ocean through

the annular gravel pack $(\mathrm{kg} / \mathrm{s})$

$Q V=$ rate of $\mathrm{CH} 4$ release from hydrate dissociation (ST

$\mathrm{m} 3 / \mathrm{s})$

$r, z=$ coordinates $(\mathrm{m})$

$r w=$ radius of the well assembly (m)

$r d 0=$ radius of the zone of complete dissociation $(\mathrm{m})$

$r d=$ outer radius of the zone of complete dissociation

(m)

rmax $=$ maximum radius of the simulation domain $(\mathrm{m})$

$S=$ phase saturation

$t=$ time (s) 
$T=$ temperature $(\mathrm{K}$ or $0 \mathrm{C})$

$V C=$ cumulative volume of $\mathrm{CH} 4$ released from hydrate

dissociation (ST m3)

$V P=$ cumulative volume of $\mathrm{CH} 4$ released into the ocean

through the annular gravel pack (ST m3)

\section{Greek Symbols}

$\alpha=$ pore compressibility (Pa-1)

$\lambda=$ van Genuchten exponent - Table 1

$\varphi=$ porosity

\section{Subscripts and Superscripts}

$0=$ denotes initial state

$\beta=$ denotes phase

$A=$ aqueous phase

$e=$ equilibrium conditions

cap $=$ capillary

$G=$ gas phase

$G 0=$ initial gas phase

$H=$ solid hydrate phase

$H O=$ initial solid hydrate phase

ir $G=$ irreducible gas

10 OTC 18193

ir $W=$ irreducible aqueous phase

$n=$ permeability reduction exponent - Table 1

$R=$ rock

$W F=$ well fluids

\section{Acknowledgment}

This work was supported by the Assistant Secretary for Fossil Energy, Office of Natural Gas and Petroleum Technology, through the National Energy Technology Laboratory, under the U.S. Department of Energy, Contract No. DEAC03-76SF00098. The authors are indebted to Stefan Finsterle, John Apps and Dan Hawkes for their thorough review and their insightful comments. Charlie Paull of the Monterey Bay Research Institute, who contributed the photograph in Figure 2, is also gratefully acknowledged.

\section{References}

1. Sloan, E.D.: Clathrate Hydrates of Natural Gases, Marcel Dekker, Inc., New York, NY (1998).

2. Durham, W.B., Kirby, S.H., Stern, L.A. and Zhang, W.: "The Strength and Rheology of Methane Clathrate Hydrate", J. Geophys. Research, 108(B4), 2182, doi:10.10292002JB001872 (2003).

3. Schmuck, E.A., and Paull, C.K.: "Evidence for Gas Accumulation Associated With Diapirism and Gas Hydrates 
at the Head of the Cape Fear Slide", Geo-Marine Letters, 13,145 (1993).

4. McIver, R.D: Hydrates of natural gas-an important agent in geologic processes, in: Abstracts with Programs, 1089, Geological Society of America (1977).

5. Paull, C.K., Buelow, W.J., Ussler W., and Borowski, W.S.: "Increased Continental Margin Slumping Frequency During Sea-Level Low Stands Above Gas Hydrate-Bearing Sediments, Geology, 24, 143 (1996).

6. Moridis, G.J., Kowalsky, M.B., and K. Pruess: "TOUGHFx/HYDRATE v1.0 User's Manual: A Code for the Simulation of System Behavior in Hydrate-Bearing Geologic Media”, Report LBNL-58950, Lawrence Berkeley National Laboratory, Berkeley, CA (2005).

7. Kim, H.C., Bishnoi, P.R., Heidemann, R.A., and Rizvi, S.S.H.: "Kinetics of Methane Hydrate Decomposition", Chem. Eng, Sci., 42(7), 1645 (1987).

8. Clarke, M.A., and Bishnoi, P.R.: "Determination of the Intrinsic Rate of Methane Gas Hydrate Decomposition", Chem. Eng. Sci., 55, 4869 (2000).

9. Aoki, Y., Shimizu, S., Yamane, T., Tanaka, T., Nakayama, K., Hayashi, T., and Okuda, Y: "Methane Hydrate Accumulation Along the Western Nankai Trough", in Gas Hydrates: Challenges for the Future. Holder, G.D., and Bishnoi, P.R., Eds., Annals of the New York Academy of Sciences, 912, 136 (2000).

10. Moridis, G.J., and Collett, T.S.: "Strategies for Gas Production From Hydrate Accumulations Under Various Geologic Conditions", Report LBNL-52568, Lawrence Berkeley Laboratory, Berkeley, CA (2003).

11. Moridis, G.J., Kowalsky, M.B., and Pruess, K.: "Depressurization- Induced Gas Production From Class 1 Hydrate Deposits", SPE 97266, 2005 SPE Annual Technical Conference and Exhibition, Dallas, Texas, U.S.A., 9 - 12 October 2005 b.

12. Wright, J.F., Dallimore, S.R., and Nixon, F.M.: "Influences of Grain Size and Salinity on Pressure-Temperature Thresholds for Methane Hydrate Stability in JAPEX/JNOC/GSC Mallik 2L-38 Gas Hydrate ResearchWell Sediments", in Scientific Results from JAPEX/JNOC/GSC Mallik 2L-38 Gas hydrate researchwell, Mackenzie Delta, Northwest Territories, Canada. Dallimore, S.R., Uchida, T., and Collett, T.S., Eds., Geological Survey f Canada Bulletin 544, 229 (1999).

13. Moridis, G.J., Seol, Y., and T. Kneafsey: "Studies of Reaction Kinetics of Methane Hydrate Dissociation in Porous Media", Report LBNL-57298, Lawrence Berkeley National Laboratory, Berkeley, CA (2005).

14. van Genuchten, M.Th.: "A Closed-Form Equation for Predicting the Hydraulic Conductivity of Unsaturated Soils”, Soil Sci. Soc., 44, 892 (1980). 


\begin{tabular}{|c|c|}
\hline \multicolumn{2}{|c|}{$\begin{array}{c}\text { Table 1 - Properties in the Reference Case } \\
\text { of the Scoping Study }\end{array}$} \\
\hline Parameter & Value \\
\hline HBS layer thickness $\Delta z$ & $1 \mathrm{~m}$ \\
\hline Initial pressure $P$ & $1.195 \times 10^{7} \mathrm{~Pa}$ \\
\hline Initial temperature $T$ & $12.9^{\circ} \mathrm{C}$ \\
\hline Initial saturations & $S_{H}=S_{A}=0.5$ \\
\hline Water salinity (mass fraction) & 0.03 \\
\hline $\begin{array}{l}\text { Hydrate-forming gas } \\
\text { composition }\end{array}$ & $100 \% \mathrm{CH}_{4}$ \\
\hline Permeability $k_{\mathrm{r}}$ & $10^{-15} \mathrm{~m}^{2}(=1 \mathrm{~m} \mathrm{D})$ \\
\hline Porosity $\phi$ & 0.30 \\
\hline Temperature of fluids at well $T_{W F}$ & $90{ }^{\circ} \mathrm{C}$ \\
\hline Grain desnity $\rho_{R}$ & $2750 \mathrm{~kg} / \mathrm{m}^{3}$ \\
\hline Dry thermal conductivity $k_{\Theta R D}$ & $1.0 \mathrm{~W} / \mathrm{m} / \mathrm{K}$ \\
\hline Wet thermal conductivity $k_{\Theta R W}$ & $3.3 \mathrm{~W} / \mathrm{m} / \mathrm{K}$ \\
\hline $\begin{array}{l}\text { Composite thermal } \\
\text { conductivity model }^{13}\end{array}$ & $\begin{array}{l}k_{\Theta C}=k_{\Theta R D} \\
+\left(S_{A}^{1 / 2}+S_{H}{ }^{1 / 2}\right) \quad\left(k_{\Theta R W}-\right. \\
\left.k_{\Theta R D}\right)+\phi S_{I} k_{\Theta I}\end{array}$ \\
\hline Capillary pressure model ${ }^{6,14}$ & $\begin{array}{l}P_{c a p}=-P_{0}\left[\left(S^{*}\right)^{-1 / \lambda}-1\right]^{\lambda} \\
S^{*}=\frac{\left(S_{A}-S_{i r A}\right)}{\left(S_{m \times A}-S_{i r A}\right)}\end{array}$ \\
\hline$S_{m \times A}$ & 1 \\
\hline$\lambda$ & 0.45 \\
\hline & 2000 \\
\hline Relative permeability model $^{6}$ & $\begin{array}{l}k_{r A}=\left(S_{A}{ }^{*}\right)^{n} \\
k_{r G}=\left(S_{G}{ }^{*}\right)^{n} \\
S_{A}{ }^{*}=\left(S_{A}-S_{i r A}\right) /\left(1-S_{i r A}\right) \\
S_{G}{ }^{*}=\left(S_{G}-S_{i r G}\right) /\left(1-S_{i r A}\right) \\
\text { OPM model }\end{array}$ \\
\hline Relative permeability exponent ${ }^{6} n$ & 4 \\
\hline & 0.02 \\
\hline & 0.20 \\
\hline
\end{tabular}


Table 2 - Well design, description and properties

\begin{tabular}{|l|c|c|c|c|c|}
\hline Component & $\begin{array}{c}\text { OD } \\
\text { radius } \\
\text { (in) }\end{array}$ & $\begin{array}{c}\text { ID } \\
\text { radius } \\
\text { (in) }\end{array}$ & $\begin{array}{c}\text { Density } \\
\left(\mathbf{k g} / \mathbf{m}^{\mathbf{3}}\right)\end{array}$ & $\begin{array}{c}\mathbf{k}_{\boldsymbol{\theta}} \\
\text { (W/m/K) }\end{array}$ & $\begin{array}{c}\mathbf{C} \\
(\mathbf{J} / \mathbf{k g} / \mathbf{K})\end{array}$ \\
\hline Conductor & 18 & 17 & 7850 & 45.35 & 461 \\
\hline Cement 1 & 17 & 10 & 1561 & 0.66 & 2100 \\
\hline Casing 1 & 10 & 9.365 & 7850 & 45.35 & 461 \\
\hline Cement 2 & 9.365 & 8 & 1561 & 0.66 & 2100 \\
\hline Casing 2 & 8 & 7.505 & 7850 & 45.35 & 461 \\
\hline Brine 1 & 7.505 & 6.6875 & 1250 & 0.55 & 4800 \\
\hline Casing 3 & 6.6875 & 6.2075 & 7850 & 45.35 & 461 \\
\hline Brine 2 & 6.2075 & 5.375 & 1250 & 0.55 & 4800 \\
\hline Casing 4 & 5.375 & 4.88 & 7850 & 45.35 & 461 \\
\hline Brine 3 & 4.88 & 2.75 & 1250 & 0.55 & 4800 \\
\hline Tubing & 2.75 & 2.389 & 7850 & 45.35 & 461 \\
\hline Hot fluids & 2.389 & - & - & - & - \\
\hline
\end{tabular}

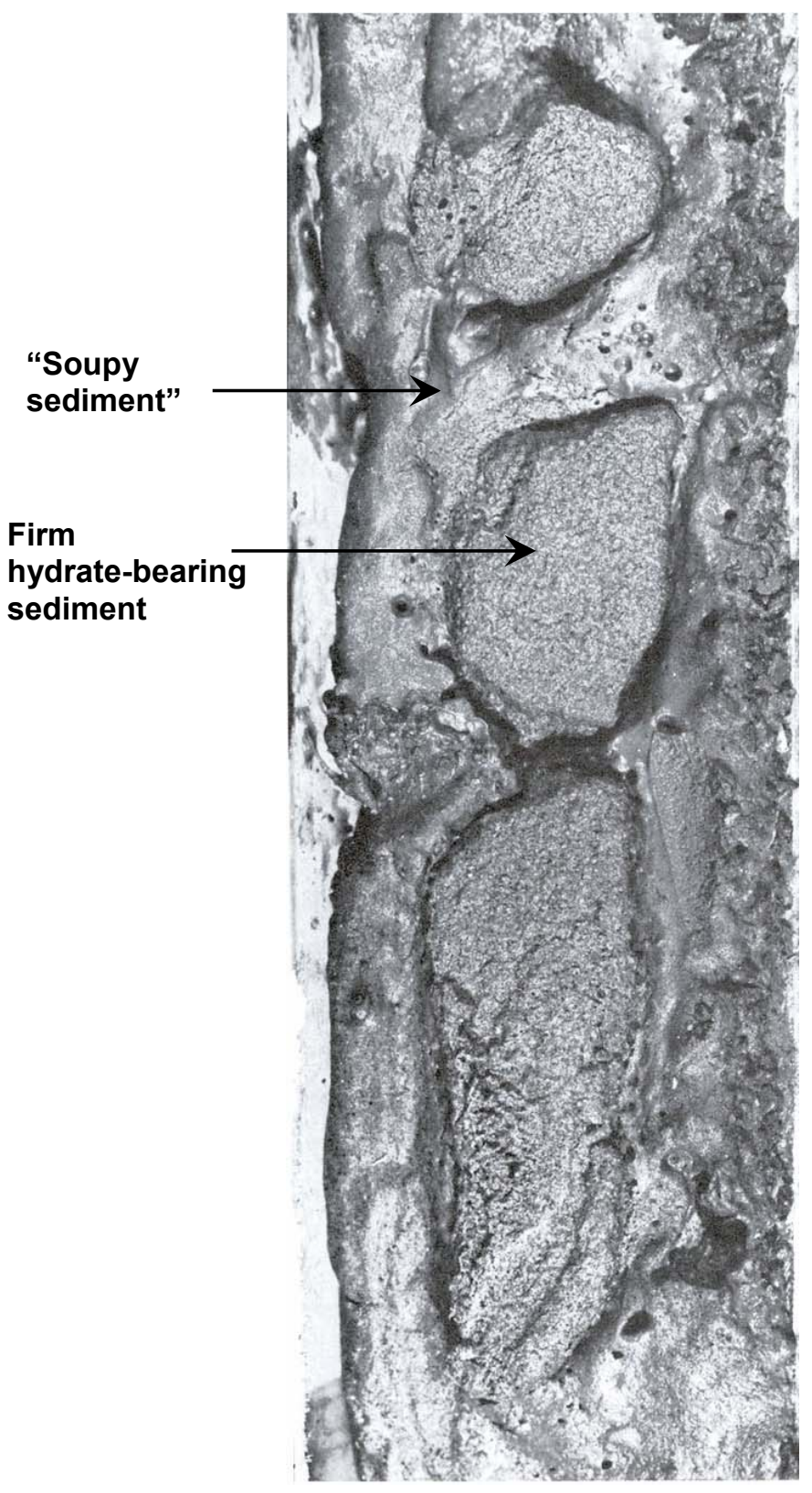

Table 3 - System description (Cases A, B, C, D)

\begin{tabular}{|c|c|c|c|c|c|}
\hline Formation & Depth (Mbml) & $\phi$ & $\mathbf{k}(\mathbf{m D})$ & $\mathbf{S}_{\mathbf{H}}$ & $\mathbf{S}_{\mathbf{A}}$ \\
\hline $\mathrm{U}$ & $0-50$ & 0 & 0 & 0 & 0 \\
\hline $\mathrm{A}$ & $50-90$ & 0.5 & $10^{-15}$ & 0.20 & 0.80 \\
\hline $\mathrm{B}$ & $90-130$ & 0.475 & $10^{-16}$ & 0.30 & 0.70 \\
\hline $\mathrm{C}$ & $130-170$ & 0.45 & $5 \times 10^{-17}$ & 0.40 & 0.60 \\
\hline $\mathrm{D}$ & $170-210$ & 0.425 & $10^{-1 /}$ & 0.45 & 0.55 \\
\hline $\mathrm{E}$ & $210-250$ & 0.40 & $5 \times 10^{-18}$ & 0.50 & 0.50 \\
\hline $\mathrm{L}$ & $250-280(\mathrm{~A}, \mathrm{~B}, \mathrm{D})$ & $0(\mathrm{~A}, \mathrm{~B}, \mathrm{D})$ & $0(\mathrm{~A}, \mathrm{~B}, \mathrm{D})$ & 0 & 0 \\
& $250-550(\mathrm{C})$ & $0.40(\mathrm{C})$ & $5 \times 10^{-18}(\mathrm{C})$ & & \\
\hline
\end{tabular}

Mbml: meters below mudline

Mudline elevation: $-1000 \mathrm{~m}$ in Cases A,C,D; $-1500 \mathrm{~m}$ in Case B

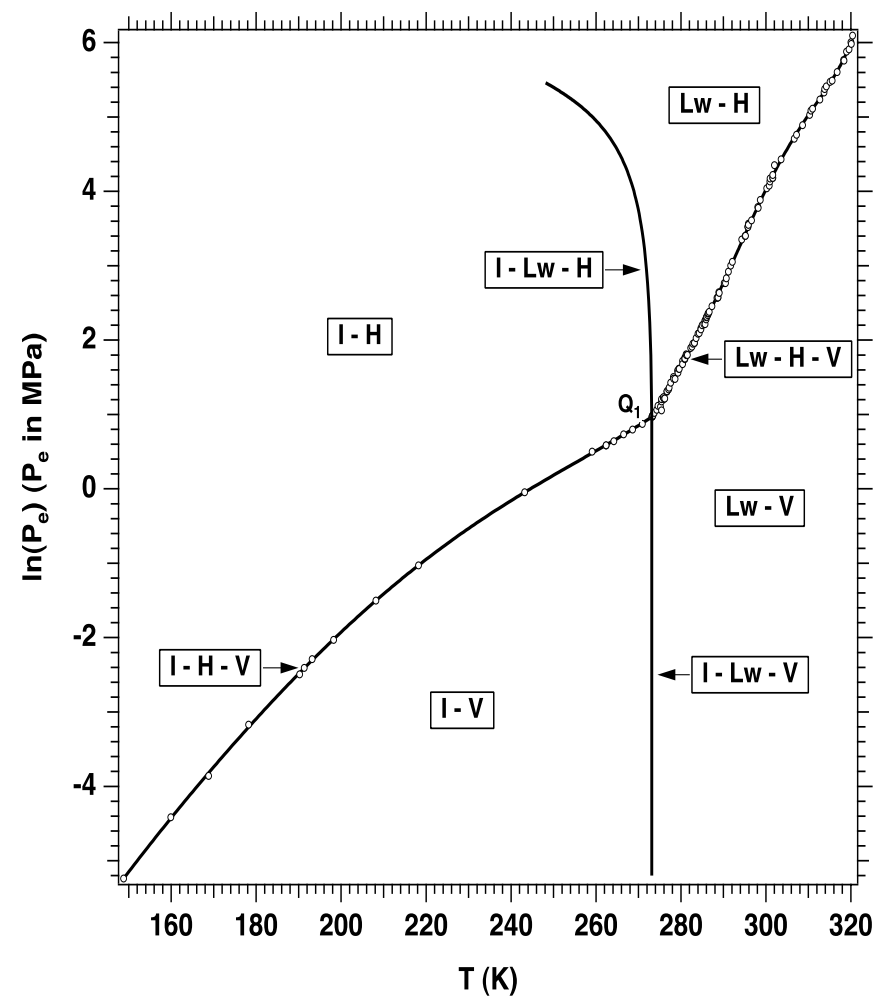

Figure 1 - Pressure-temperature equilibrium relationship in the phase diagram of the water-- $\mathrm{CH}_{4}-$-hydrate system (Moridis et al. ${ }^{6}$ ).

Figure 2 - Dissociating sample of a marine HBS core. Note the firm appearance of the hydrate-bearing inner core, as opposed to the "soupy sediment" (soft mud of fluid consistency with evidence of bubbling gas) in the dissociating outer annulus (source: Deep Sea Drilling Program, Leg 67, courtesy of C.K Paull of the Monterey Bay Research Institute). 


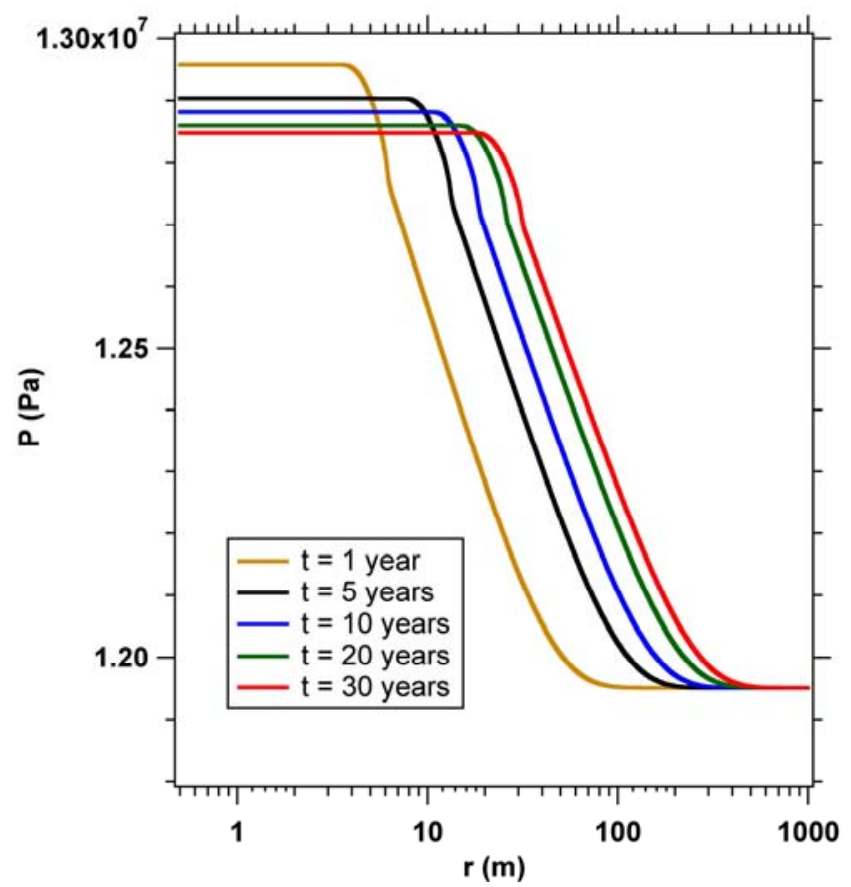

Figure 3 - Spatial distribution of pressure $P$ in the reference case of the 1D scoping studies.

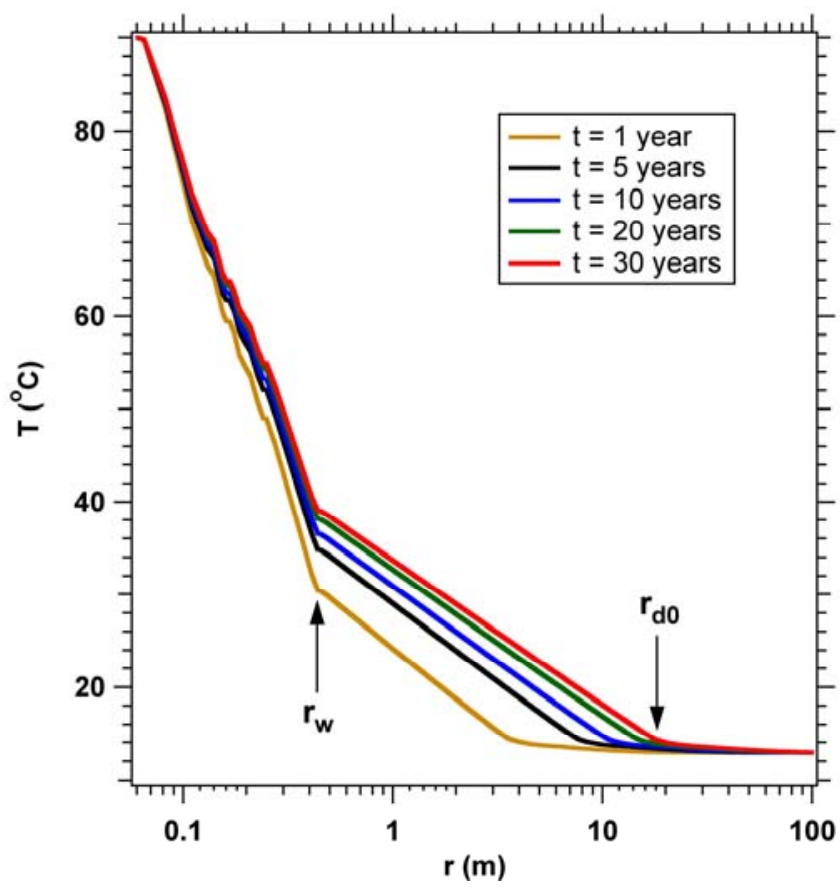

Figure 4 -Spatial distributions of temperature $T$ in the reference case of the 1D scoping studies. Note that (a) the radius of the wellbore assembly $\left(r_{w}-\right.$ static) and (b) the radius of the zone of complete dissociation ( $r_{d 0}-$ evolving with time) are identified by the abrupt change in slope of the T distribution.
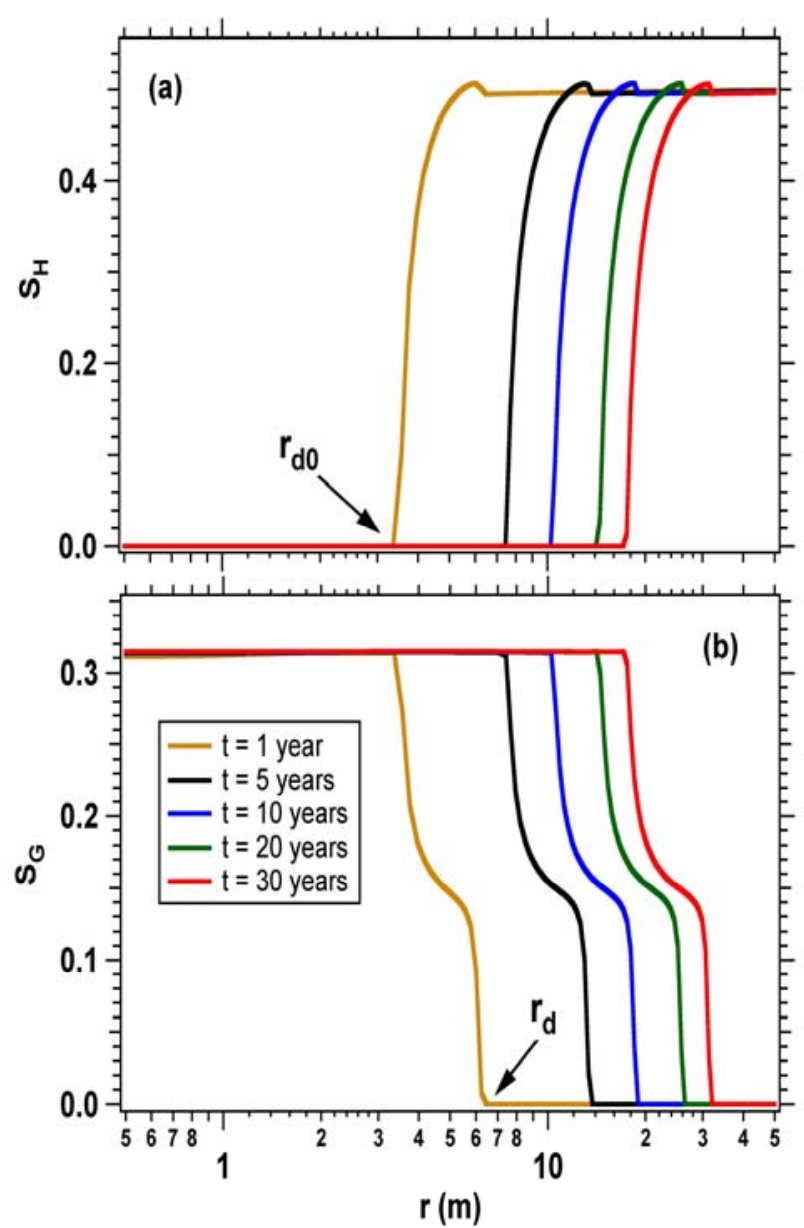

Figure 5 - Spatial distributions of (a) $S_{H}$, and (b) $S_{G}$ in the reference case of the 1D scoping studies. The radius of the zone of complete dissociation $\left(r_{d 0}\right)$ and the radius of the active zone of dissociation $\left(r_{d}\right)$ are identified (they evolve with time). 

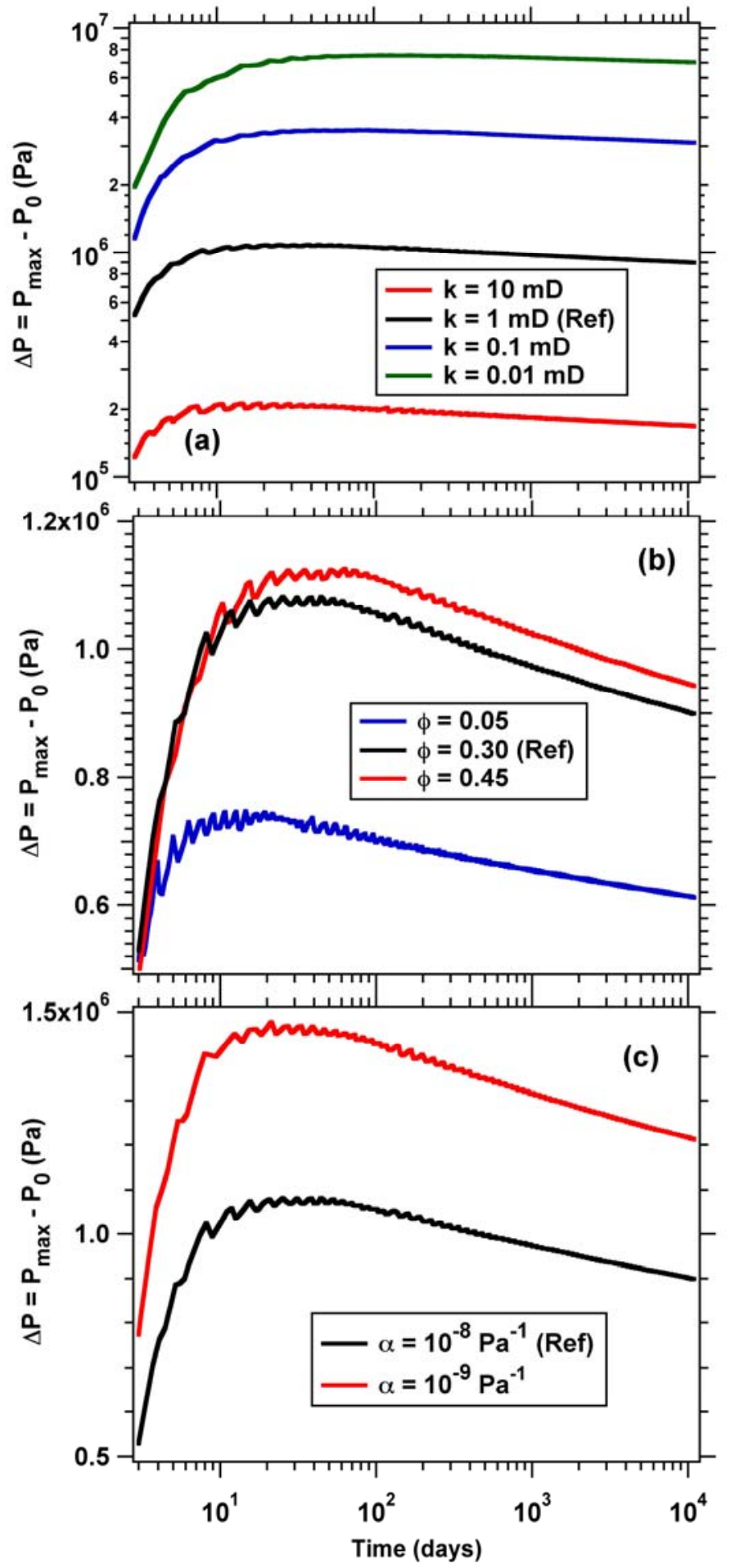

Figure 6 - Scoping studies: sensitivity of $\boldsymbol{P}_{\max }$ (described as $\Delta P=$ $P_{\max }-P_{0}$ ) to variations in (a) intrinsic permeability $k$, (b) porosity $\phi$, and (c) pore compressibility $\alpha$.
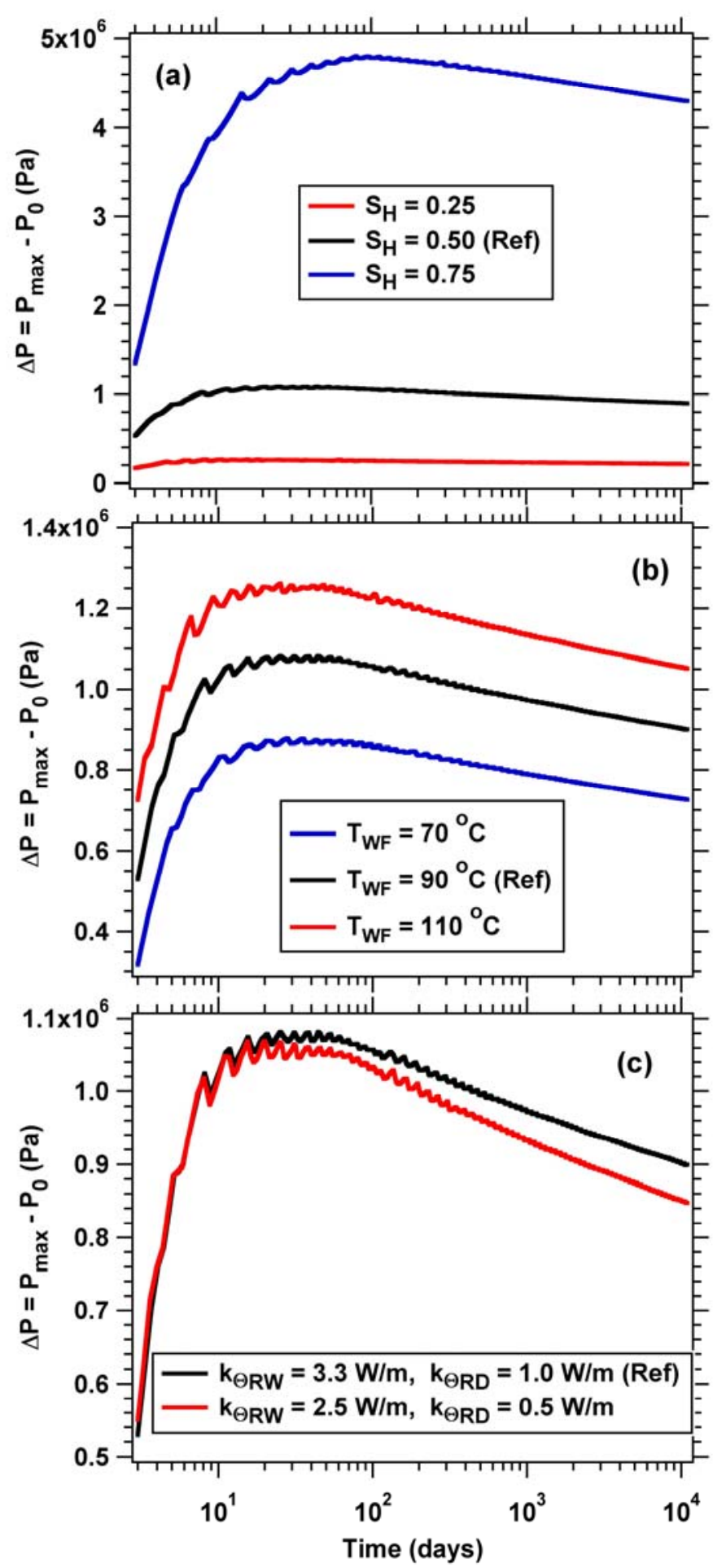

Figure 7 - Scoping studies: sensitivity of $P_{\max }$ (described as $\Delta P=$ $P_{\max }-P_{0}$ ) to variations in (a) the hydrate saturation $S_{H}$, (b) the temperature of well fluids $T_{W F}$, and (c) the medium thermal conductivities. 

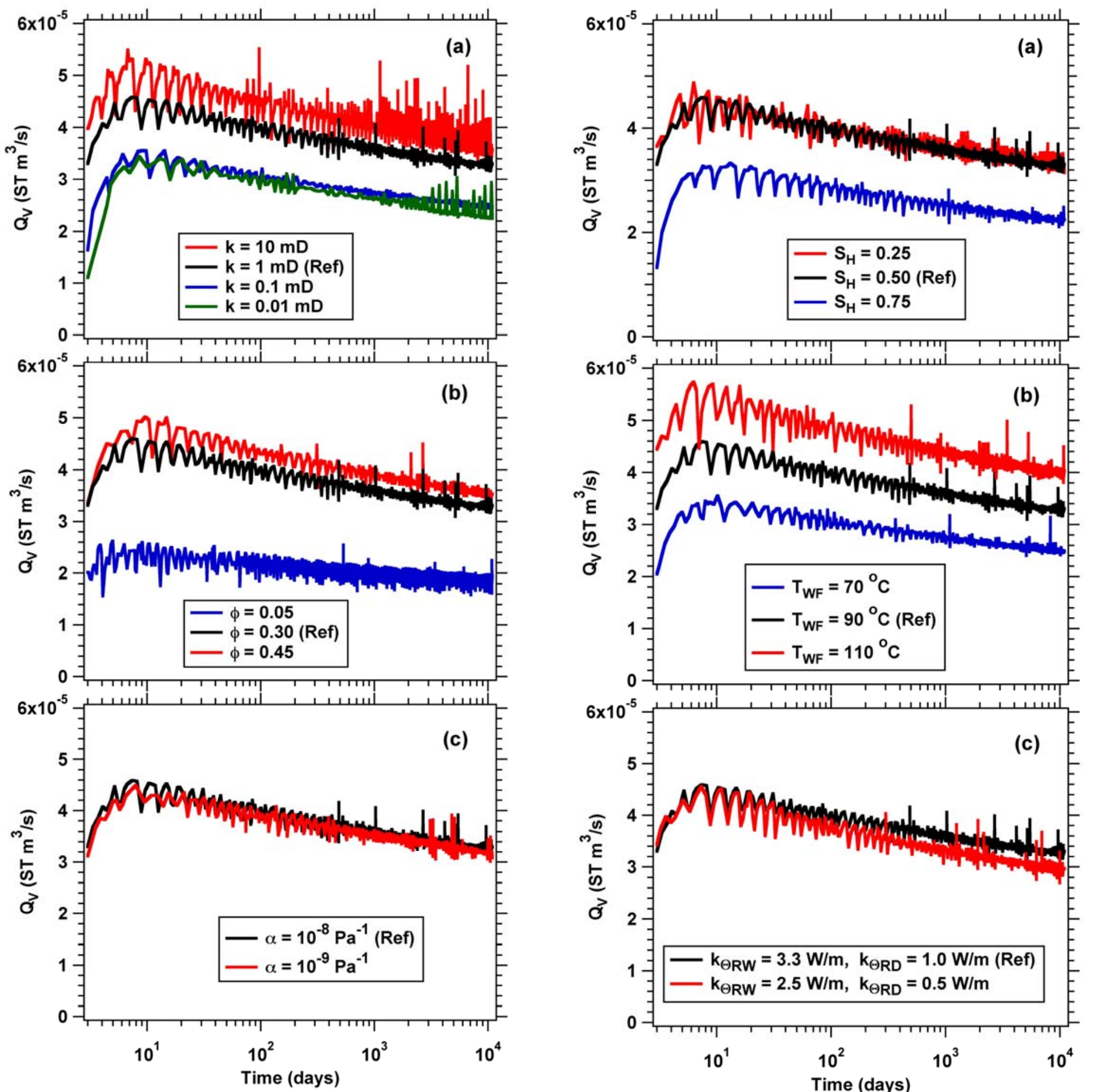

Figure 8 - Scoping studies: sensitivity of the rate of gas release from hydrate dissociation $Q_{V}$ to variations in (a) intrinsic permeability $k$, (b) porosity $\phi$, and (c) pore compressibility $\alpha$.

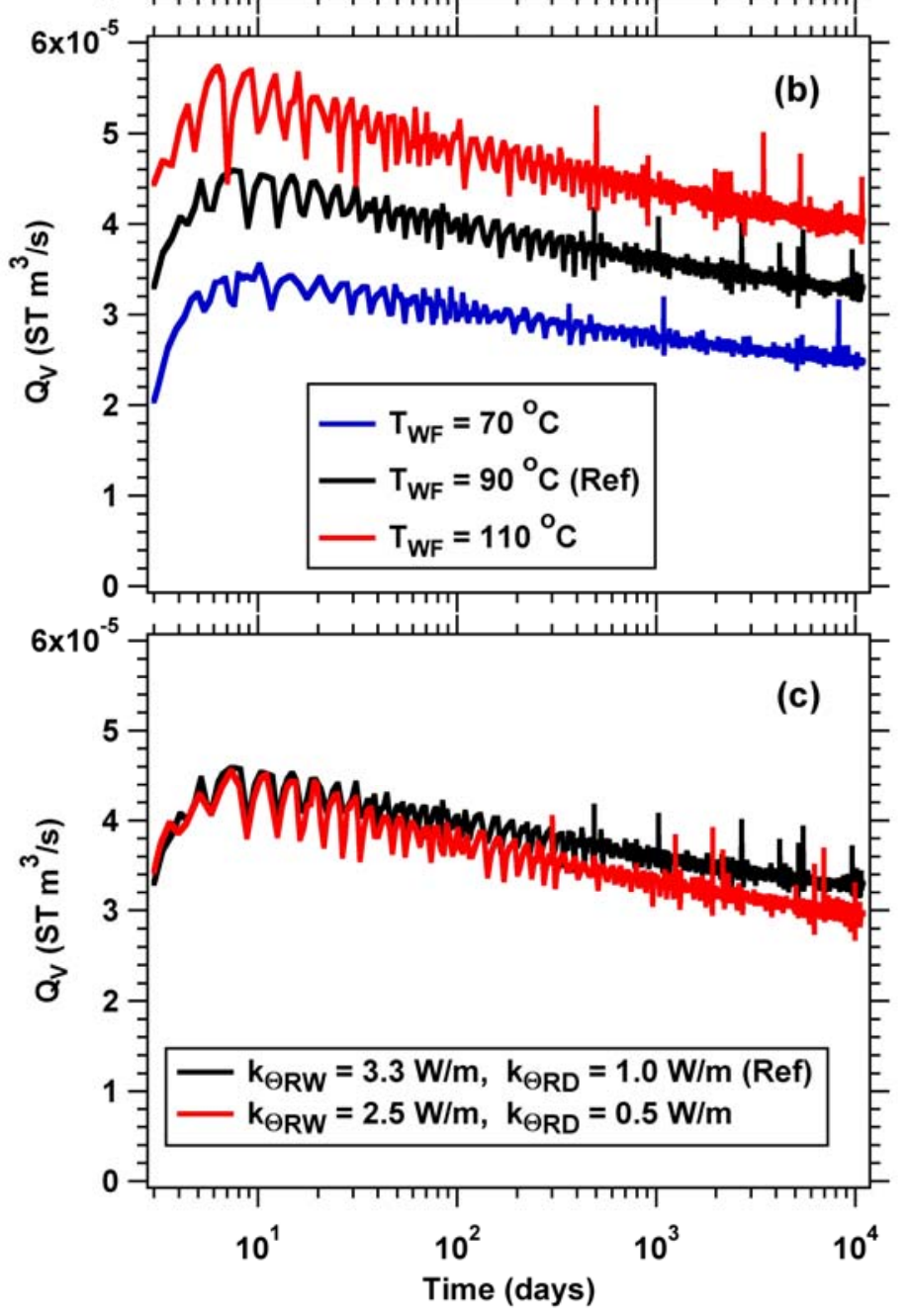

Figure 9 - Scoping studies: sensitivity of the rate of gas release from hydrate dissociation $Q_{V}$ to variations in (a) the hydrate saturation $S_{H}$, (b) the temperature of well fluids $T_{W F}$, and (c) the medium thermal conductivities. 

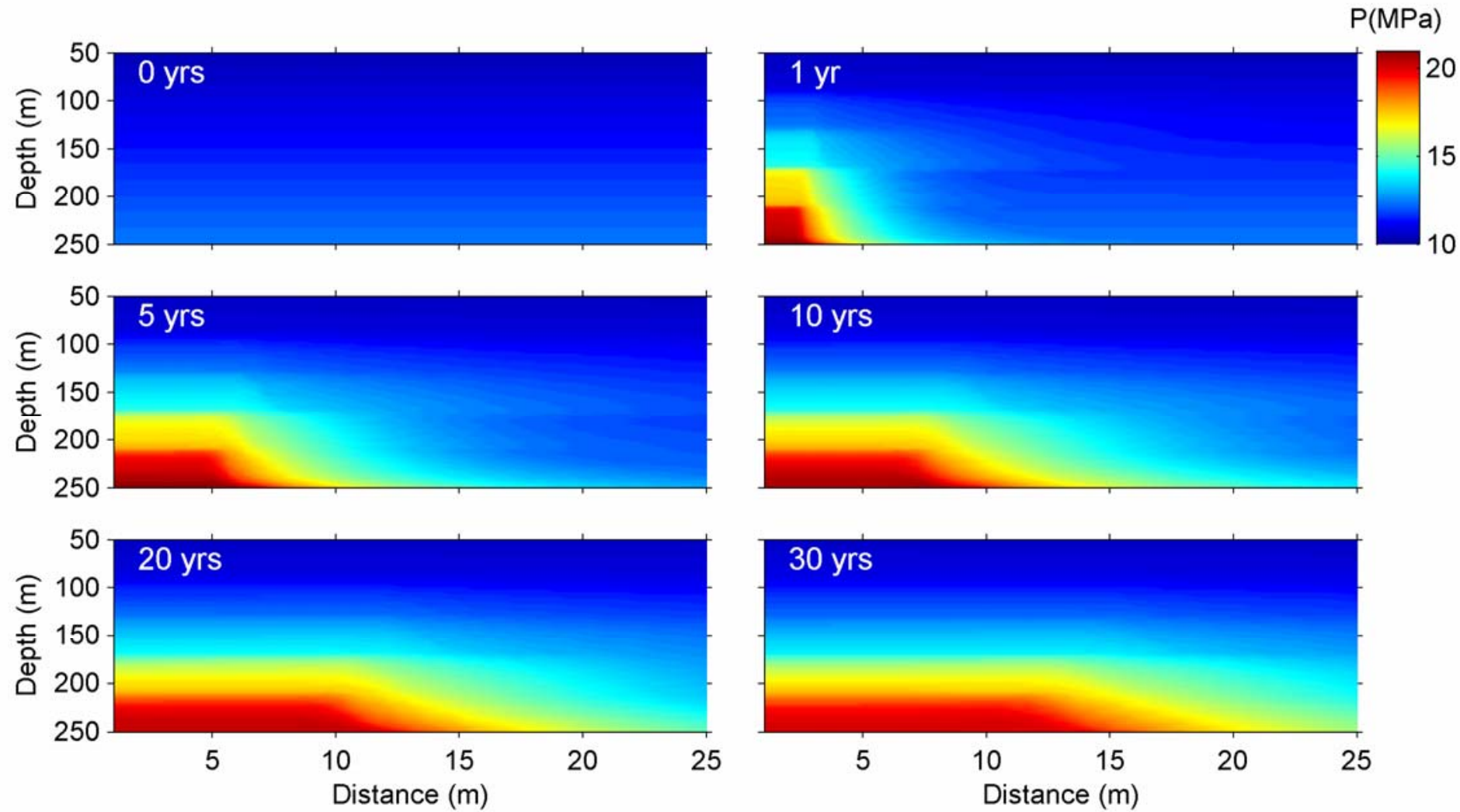

Figure 10. Evolution of spatial distribution of pressure in the HBS of Case A (reference case).
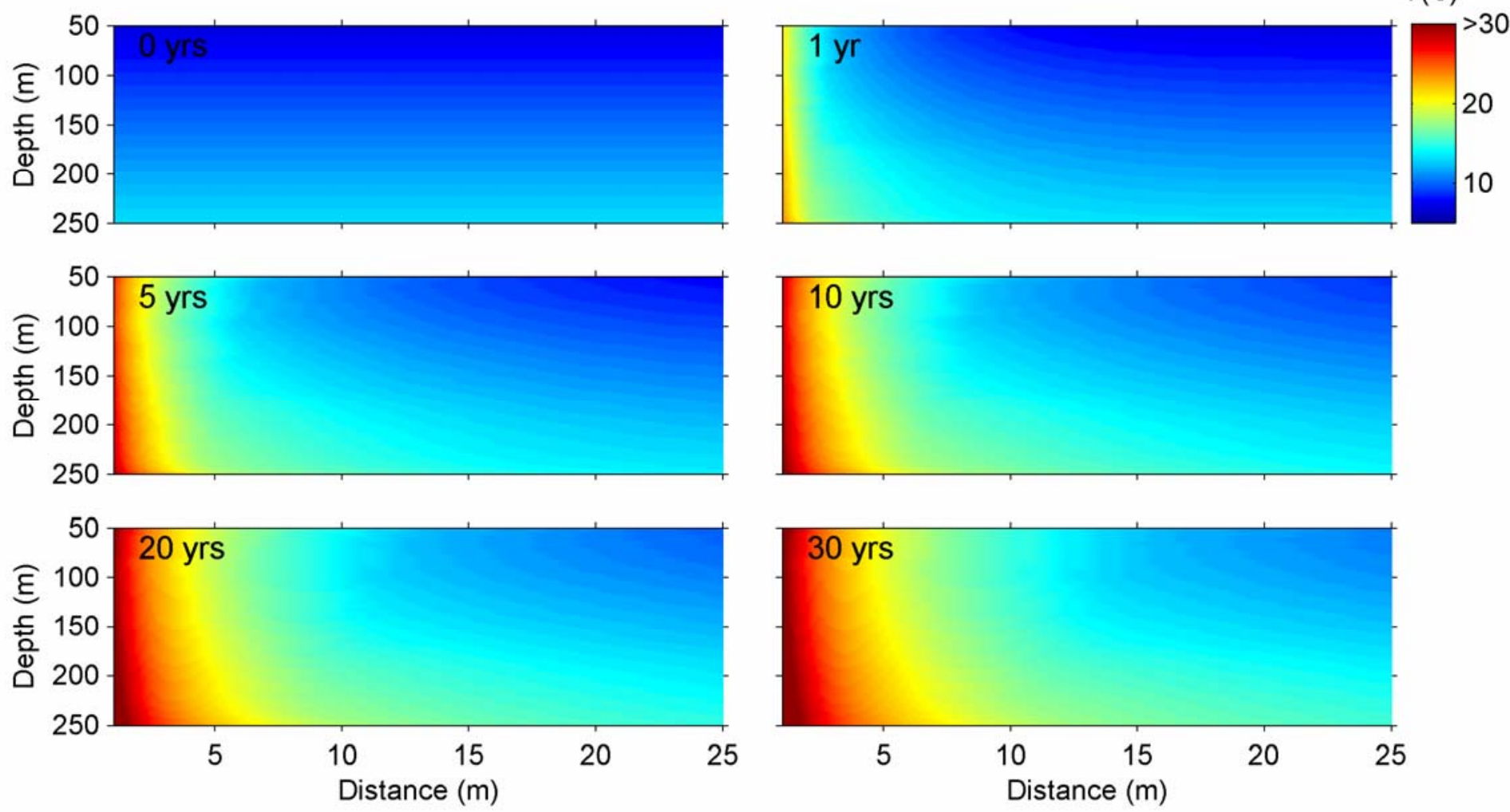

Figure 11. Evolution of spatial distribution of temperature in the HBS of Case A (reference case). 

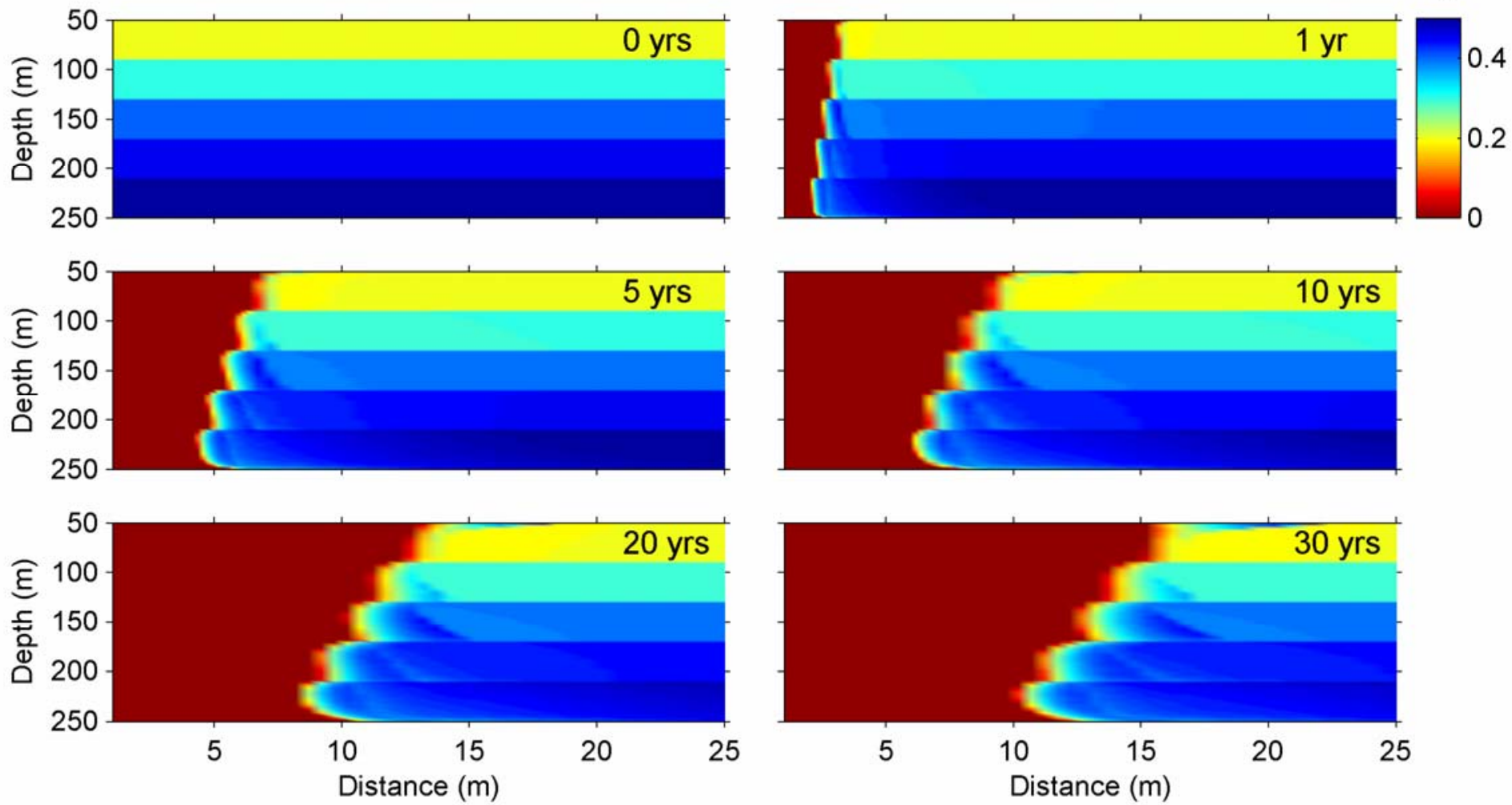

Figure 12. Evolution of spatial distribution of $S_{H}$ in the HBS of Case $A$ (reference case).
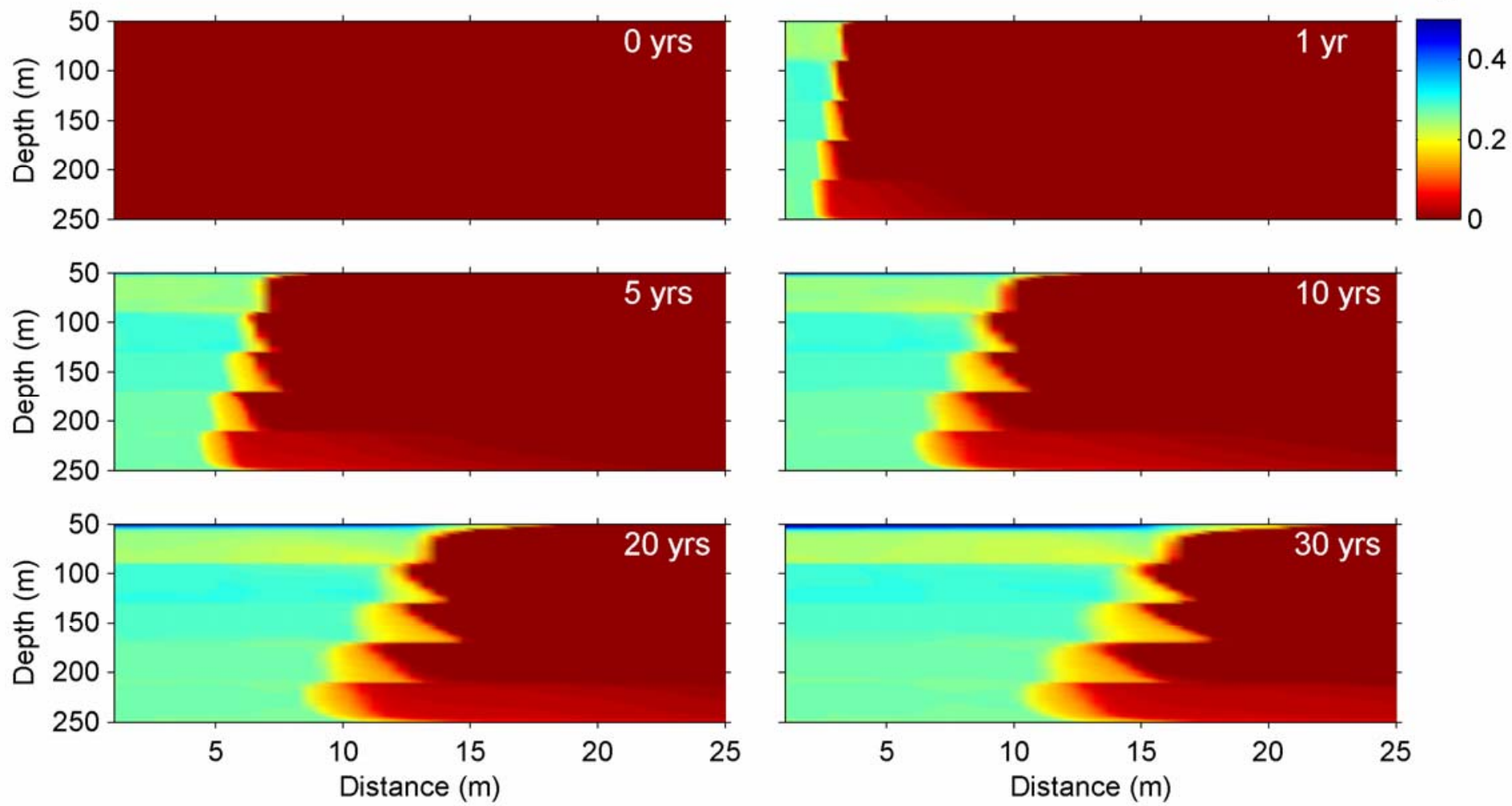

Figure 13. Evolution of spatial distribution of $S_{G}$ in the HBS of Case $A$ (reference case). 

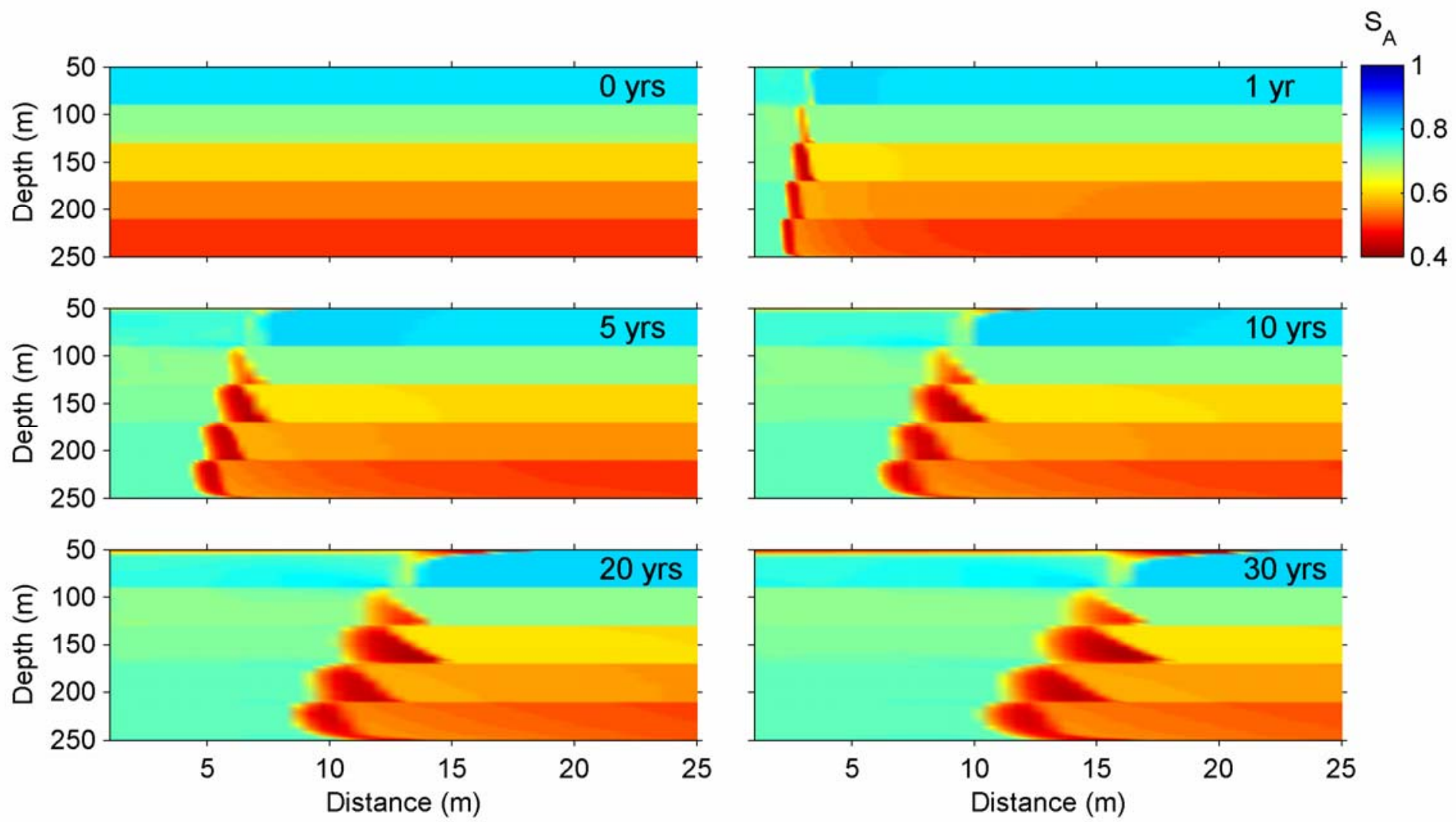

Figure 14. Evolution of spatial distribution of $S_{A}$ in the HBS of Case $A$ (reference case).
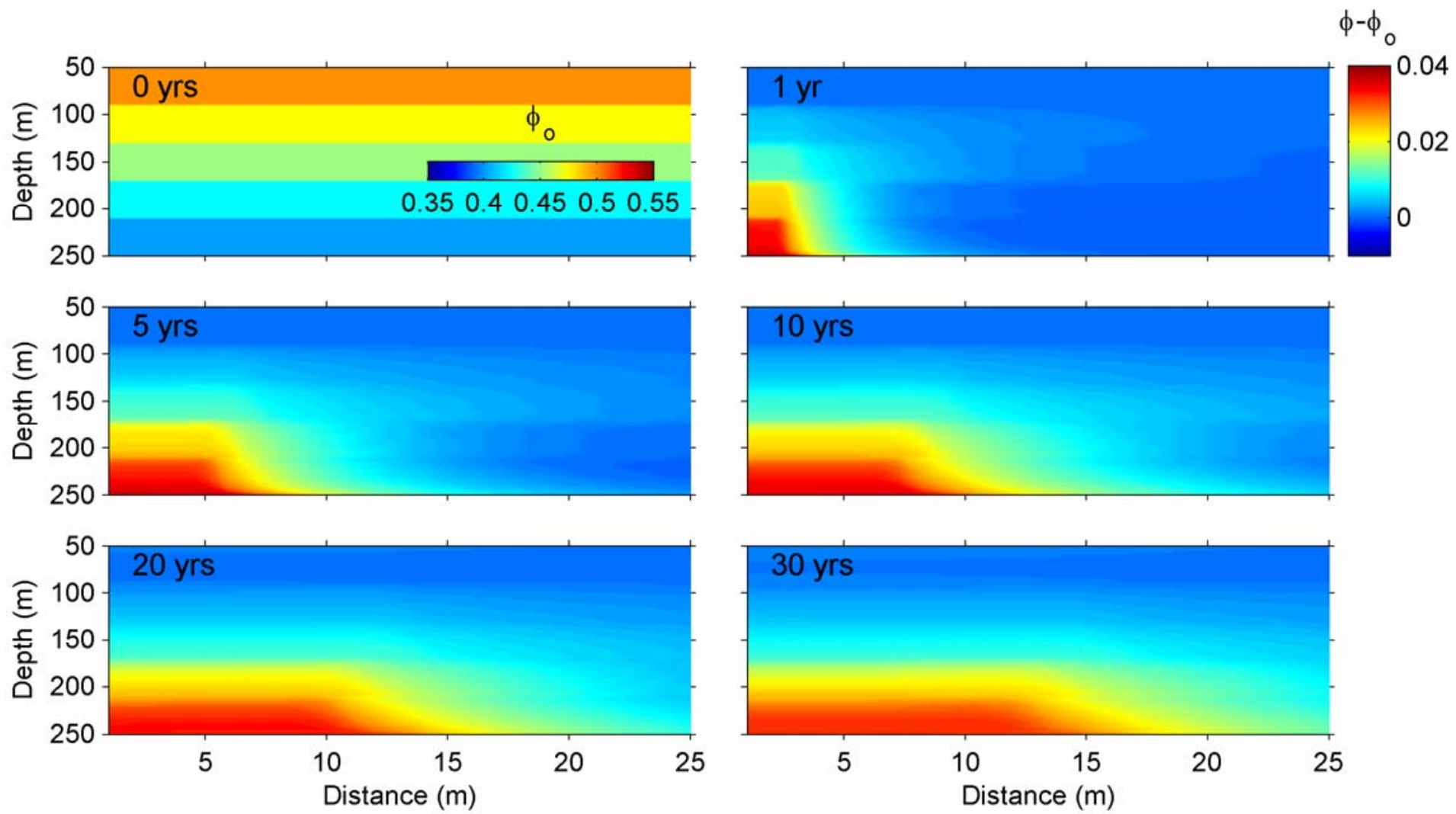

Figure 15. Evolution of spatial distribution of porosity deviation $\Delta \phi=\phi-\phi_{0}$ in the HBS of Case A (reference case). 

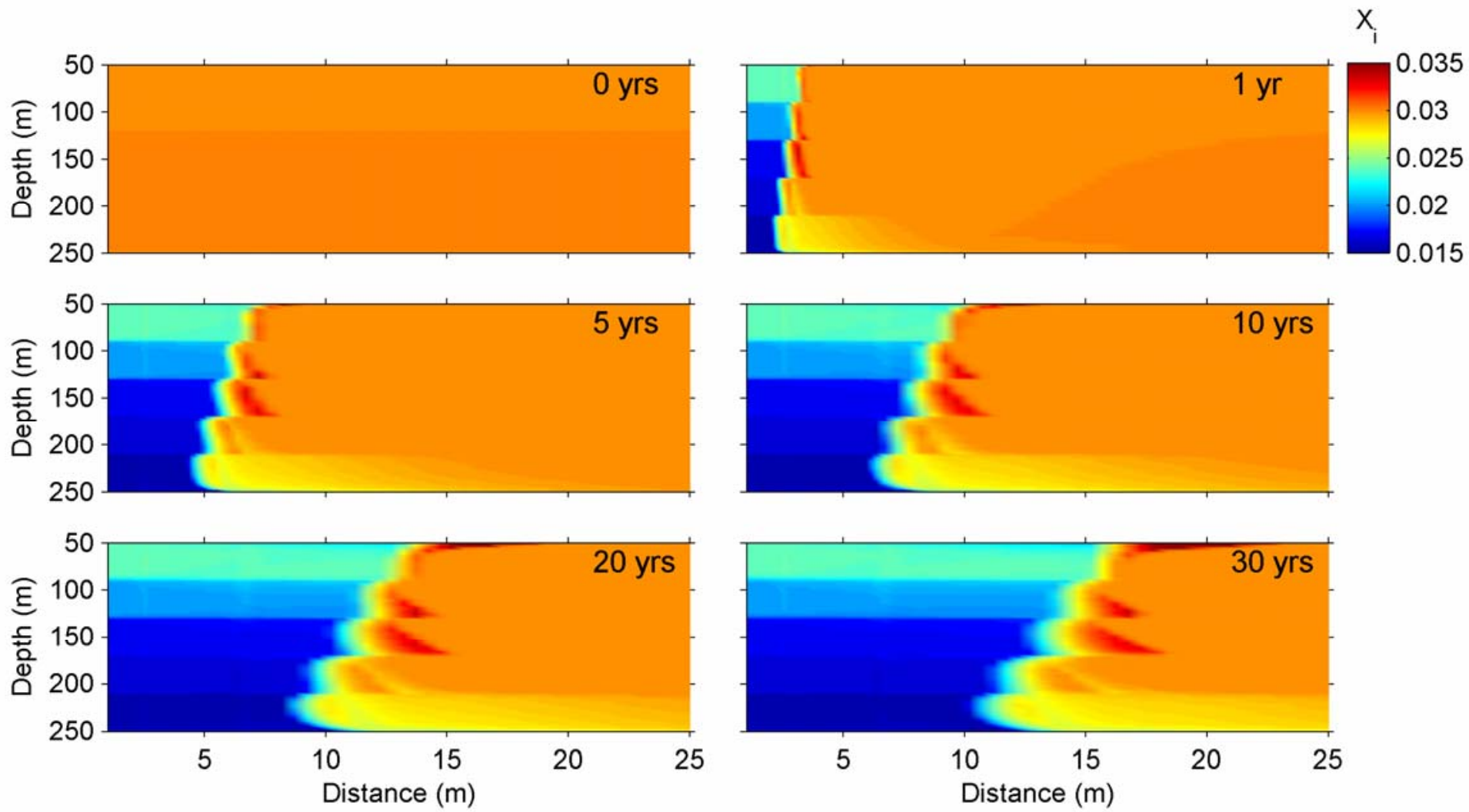

Figure 16. Evolution of spatial distribution of the salt mass fraction in the aqueous phase in Problem M2 (reference case) at Location \#2.

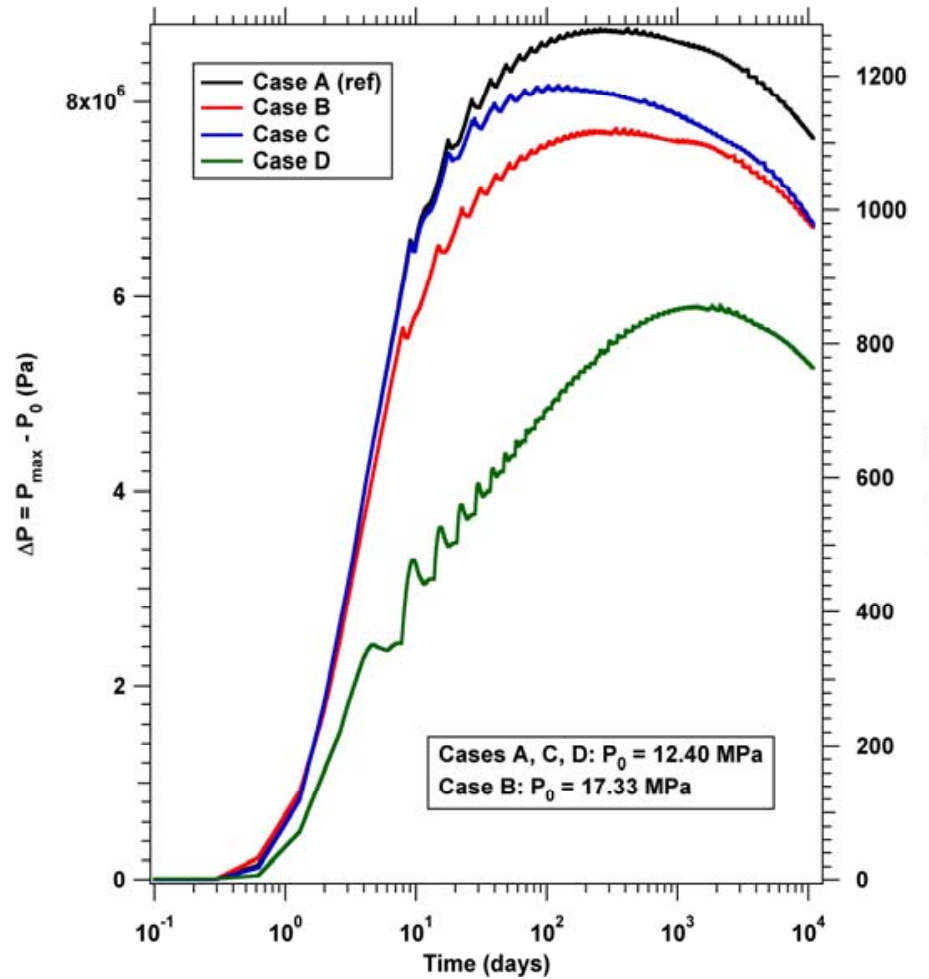

Figure 17. Evolution of maximum pressure in the hydrate-bearing sediments in Cases A through D.

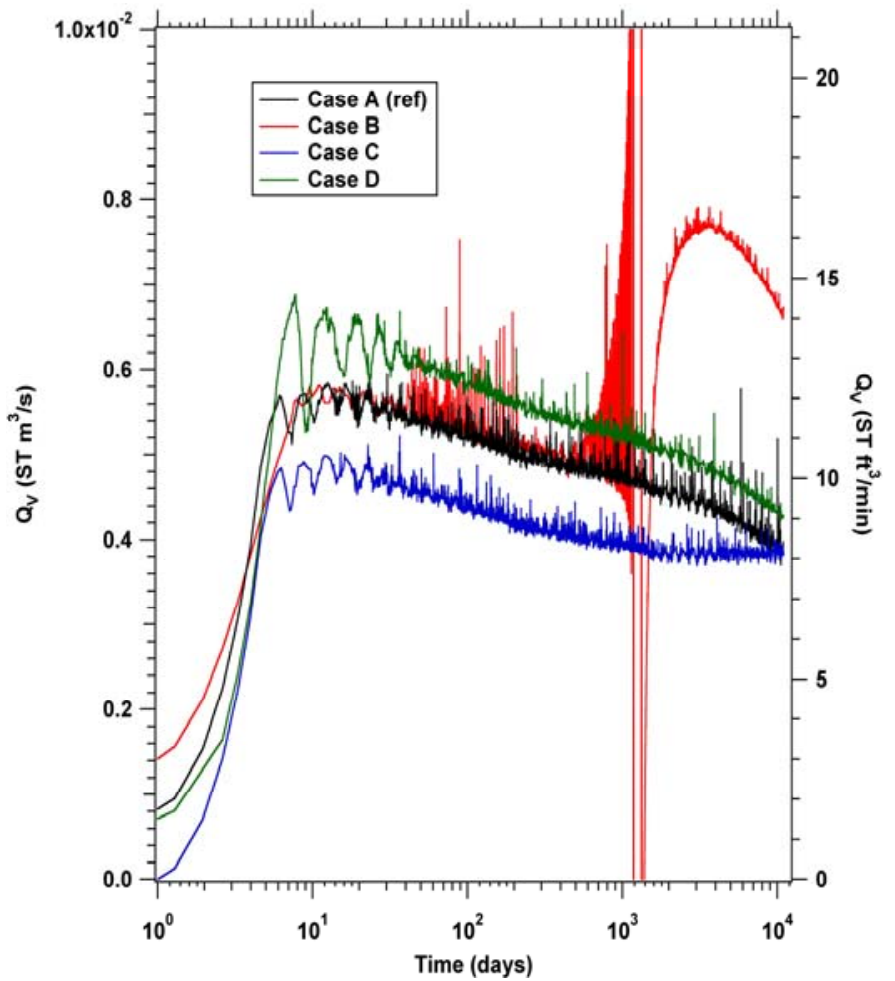

Figure 18. Volumetric rate $Q_{v}$ of release of $\mathrm{CH}_{4}$ originating from hydrate dissociation (Cases A through D). 


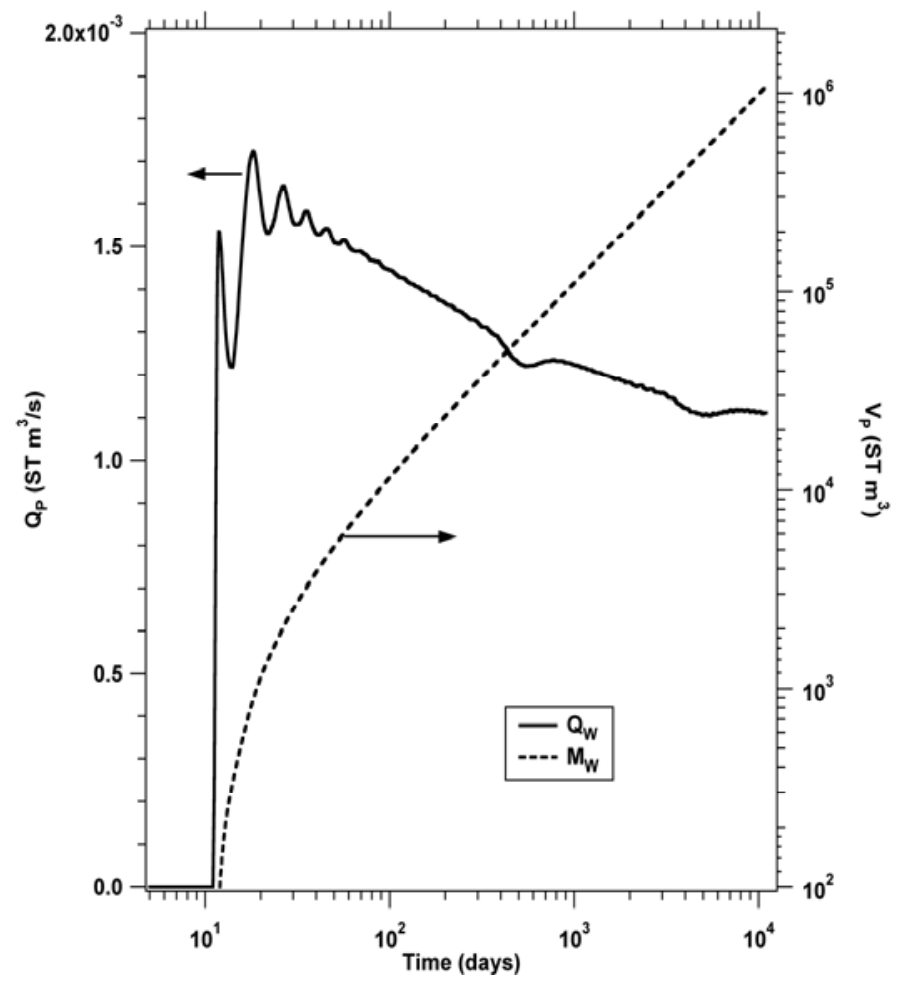

Figure 19. Case D: Evolution of volumetric rate of gas production $\left(Q_{P}\right)$, and cumulative volume of gas produced from the gravel pack $\left(V_{P}\right)$.

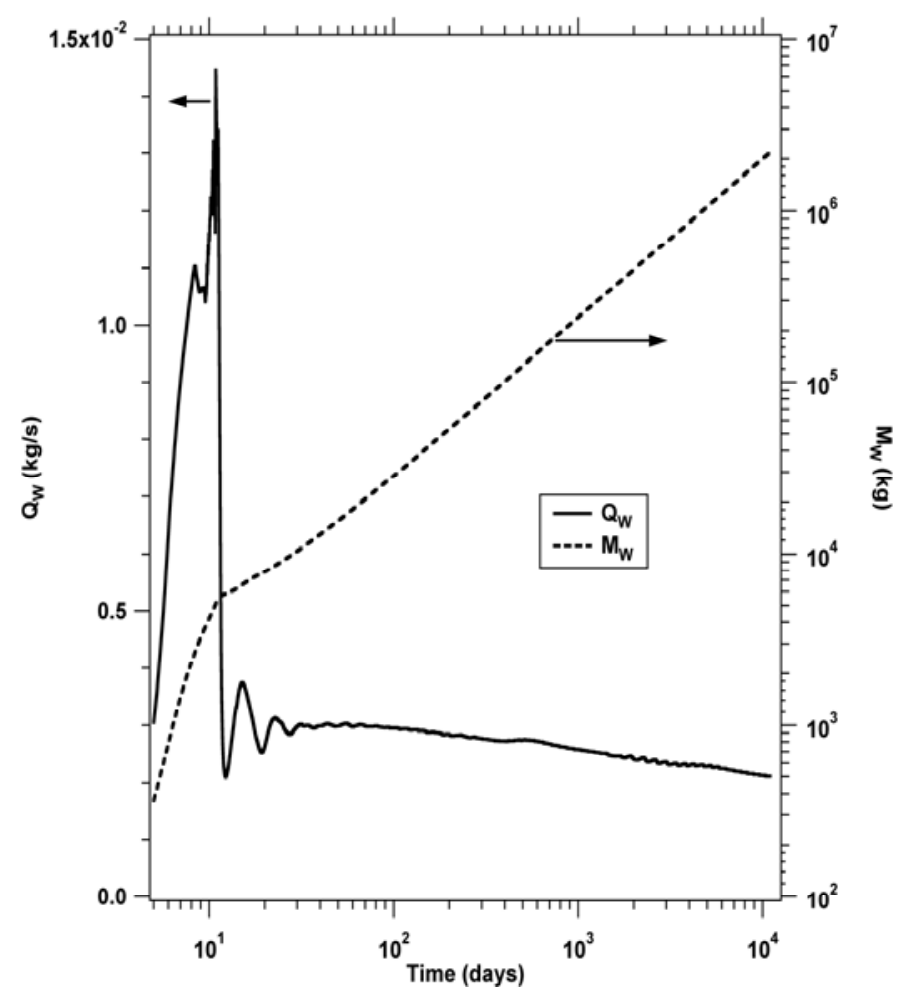

Figure 20. Case D: Evolution of mass rate of water production $\left(Q_{w}\right)$, and cumulative mass of water produced from the gravel pack $\left(\mathrm{M}_{\mathrm{w}}\right)$.

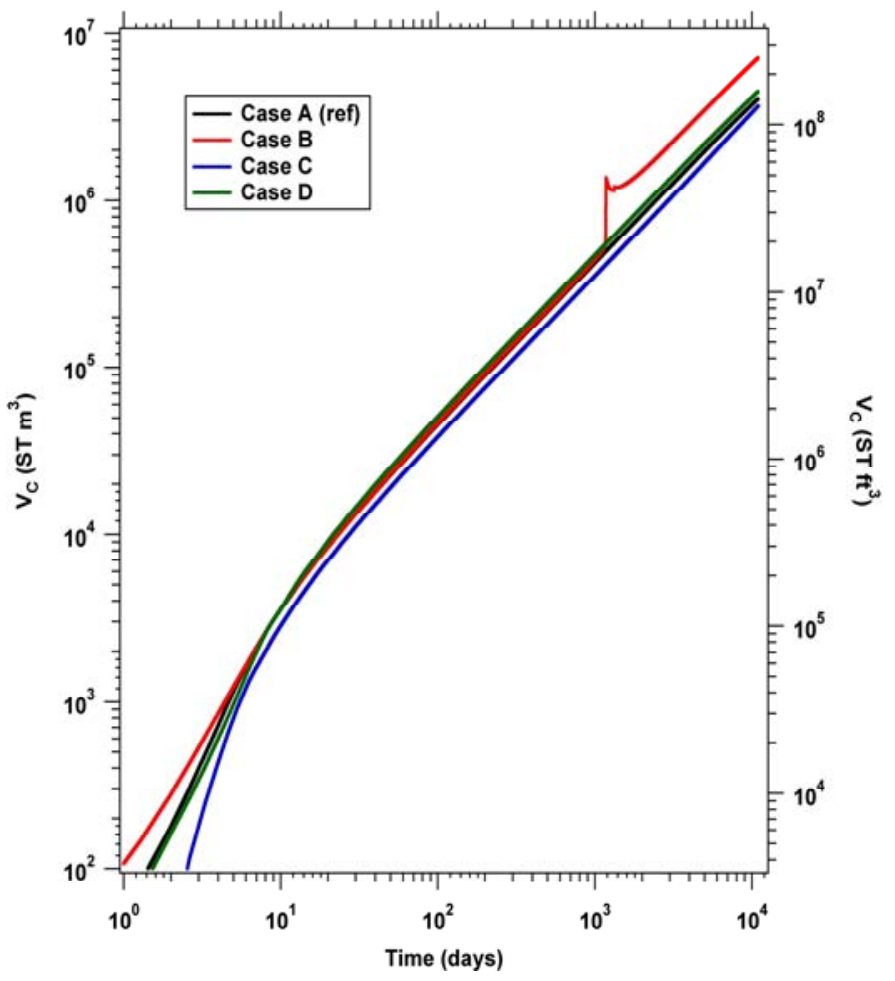

Figure 21. Cumulative volume of $\mathrm{CH}_{4}$ released from hydrate dissociation in Cases A through D. 